\title{
Pseudoscalar Goldstone bosons in the color-flavor locked phase at moderate densities
}

\author{
Verena Kleinhaus, ${ }^{1}$ Michael Buballa, ${ }^{1}$ Dominik Nickel,${ }^{1}$ and Micaela Oertel ${ }^{2}$ \\ ${ }^{1}$ Institut für Kernphysik, Technische Universität Darmstadt, Germany \\ ${ }^{2}$ LUTH, Observatoire de Paris, CNRS, Université Paris Diderot, 5 place Jules Janssen, 92195 Meudon,France
}

(Dated: August 17, 2021)

\begin{abstract}
The properties of the pseudoscalar Goldstone bosons in the color-flavor locked phase at moderate densities are studied within a model of the Nambu-Jona-Lasinio type. The Goldstone bosons are constructed explicitly by solving the Bethe-Salpeter equation for quark-quark scattering in random phase approximation. Main focus of our investigations are (i) the weak decay constant in the chiral limit, (ii) the masses of the flavored (pseudo-) Goldstone bosons for non-zero but equal quark masses, (iii) their masses and effective chemical potentials for non-equal quark masses, and (iv) the onset of kaon condensation. We compare our results with the predictions of the low-energy effective field theory. The deviations from results obtained in the weak-coupling limit are discussed in detail.
\end{abstract}

\section{INTRODUCTION}

Much effort has recently been devoted to the study of strongly interacting matter at nonzero baryon density. In particular the rich phase structure of color superconducting quark matter has attracted much interest. (For reviews on color superconductivity see, e.g., Refs. [1-8].) In nature, quark matter phases might be realized in compact stars [9-11]. It is therfore natural to ask whether quark pairing has interesting phenomenological consequences for compact star physics. In this context the energetically lowest lying degrees of freedom are relevant for many dynamic properties of quark matter.

At low temperatures and very high densities the preferred state is most probably the color-flavor locked(CFL) phase where up, down, and strange quarks are paired in a particularly symmetric way [12]. This can be shown from first principles within a weak-coupling expansion [13-15]. Although this expansion is not valid at "moderate" densities which could be reached in compact stars, recent Dyson-Schwinger studies indicate that the CFL phase might be the preferred phase all the way down to the hadronic phase [16].

In the CFL phase, all quark flavors and colors participate in a condensate. As a consequence, all fermionic modes are gapped and do not appear in the low-energy excitation spectrum. The diquark condensates break the original $U(1)_{\text {baryon }} \times S U(3)_{\text {color }} \times S U(3)_{L} \times S U(3)_{R}$ symmetry of three-flavor QCD (in the chiral limit) down to a residual $Z_{2} \times S U(3)_{c o l o r}+V$, corresponding to a simultaneous ("locked") rotation in color and flavor space. Due to the breaking of the color symmetry, all eight gluons receive a mass, while the breaking of baryon number and chiral symmetry leads to the emergence of one scalar and eight pseudoscalar Goldstone bosons. In addition, there is a ninth pseudoscalar Goldstone boson related to the spontaneous breaking of $U_{A}(1)$ which is a symmetry of QCD at very high density $[17,18]$. In the presence of quark masses chiral symmetry is broken explicitly and the pseudoscalar Goldstone bosons aquire a mass, while the scalar Goldstone boson remains massless. Since, with all quarks being gapped, the Goldstone bosons are the lowest lying excitations, they play an important role for the thermodynamic and transport properties of strongly interacting matter, relevant for compact star phenomenology (cf., e.g., Refs. [19-21]).

The symmetry breaking pattern is the basis for the construction of the low-energy effective theory (LEET) [22-28], which describes the Goldstone boson dynamics and is valid for energies much smaller than the superconducting gap. At very high densities, the interaction is weak and the constants for the LEET can be calculated from QCD using High Density Effective Theory (HDET) [26, 29-31]. For instance, in the weak-coupling limit, pseudoscalar meson masses and decay constants have been investigated $[23,30,32-35]$. It was also shown that the stress imposed by the strange quark mass on the CFL Cooper pairs acts as an effective strangeness chemical potential, which may eventually lead to kaon condensation $[24,26,27]$.

At intermediate densities, relevant for compact star phenomenology, the interaction becomes nonperturbative and it is difficult to study the Goldstone boson dynamics from first principles. The leading-order predictions, however, are often universal, in the sense that they do not depend on the interaction, but should hold in any model exhibiting the same symmetry pattern. One such model is the Nambu-Jona-Lasinio (NJL) model [36], often used in the intermediate density regime to study at least qualitatively the main features. (For reviews, see, e.g., Refs. [5, 37-39].) This model has already been applied to study kaon condensation in the CFL phase at non-zero strange quark masses [40-42]. However, this was done by focusing on the ground state properties, i.e., without explicit construction of the Goldstone bosons. In Refs. [43, 44], on the other hand, meson and diquark properties in the CFL phase have been studied explicitly, but this investigation was restricted to the chiral limit. (Mesons and diquarks in the 2SC phase have been discussed in Refs. [45, 46].)

In the present paper we discuss a detailed analysis of 
properties of pseudoscalar mesons ${ }^{1}$ in the CFL phase in an NJL-type model including the cases of equal and unequal nonzero quark masses. Emphasis is put on a comparison with the weak-coupling results.

The paper is organized as follows. In Sec. II we introduce our model and discuss how to construct the mesonic excitations. Sec. III is devoted to some general results which can be obtained in the limit of equal quark masses based on chiral Ward-Takahashi identities. In Sec. IV numerical results will be presented. Within that section, we investigate the pion decay constant in the chiral limit as well as meson masses for the cases of equal and unequal quark masses. In this context we also discuss the onset of kaon condensation in the CFL phase. Our results are summarized in Sec. V.

\section{FORMALISM}

\section{A. Model Lagrangian}

We consider an NJL-type Lagrangian

$$
\mathcal{L}=\bar{q}(i \not \partial-\hat{m}) q+\mathcal{L}_{q q},
$$

where $q$ is a quark field with three flavor and three color degrees of freedom, $\hat{m}=\operatorname{diag}_{f}\left(m_{u}, m_{d}, m_{s}\right)$ is the mass matrix, and

$$
\begin{aligned}
& \mathcal{L}_{q q}=H \sum_{A, A^{\prime}=2,5,7}\left[\left(\bar{q} i \gamma_{5} \tau_{A} \lambda_{A^{\prime}} C \bar{q}^{T}\right)\left(q^{T} C i \gamma_{5} \tau_{A} \lambda_{A^{\prime}} q\right)\right. \\
& \left.+\left(\bar{q} \tau_{A} \lambda_{A^{\prime}} C \bar{q}^{T}\right)\left(q^{T} C \tau_{A} \lambda_{A^{\prime}} q\right)\right]
\end{aligned}
$$

describes an $S U(3)_{\text {color }} \times U(3)_{L} \times U(3)_{R}$ symmetric fourpoint interaction with a dimensionful coupling constant $H$. $C=i \gamma^{2} \gamma^{0}$ is the matrix of charge conjugation, and $\tau$ and $\lambda$, denote Gell-Mann matrices acting in flavor space and color space, respectively. In this article, we follow the convention that the indices $A$ and $A^{\prime}$ are used for the antisymmetric Gell-Mann matrices only, i.e., $A, A^{\prime} \in$ $\{2,5,7\}$, whereas arbitrary Gell-Mann matrices will be denoted by small Latin letters, e.g., $\tau_{a}, a=1, \ldots, 8$.

The first term in Eq. (2) corresponds to a scalar quarkquark interaction in the color and flavor antitriplet channel, just as needed for giving rise to the diquark condensates in the CFL phase (see Eq. (12) below). The second term is the corresponding pseudoscalar interaction and is required by chiral symmetry. This will be the essential term for the pseudoscalar Goldstone excitations we want to study.

For simplicity, we restrict ourselves to quark-quark interactions. The effect of quark-antiquark interactions, which give rise to normal self-energies and thereby to dynamical quark masses, will be investigated in a future publication.

\footnotetext{
${ }^{1}$ In this article, we often use the words "mesons" and "diquarks" synonymously, see Sec. II E for more details.
}

\section{B. Operators in Nambu-Gorkov space}

Introducing Nambu-Gorkov bispinors,

$$
\Psi=\frac{1}{\sqrt{2}}\left(\begin{array}{c}
q \\
C \bar{q}^{T}
\end{array}\right)
$$

Eq. (2) can be rewritten as

$$
\begin{aligned}
& \mathcal{L}_{q q}=4 H \sum_{A, A^{\prime}=2,5,7}\left\{\bar{\Psi} \Gamma_{A A^{\prime}}^{s \uparrow} \Psi \bar{\Psi} \Gamma_{A A^{\prime}}^{s \downarrow} \Psi\right. \\
& \left.+\bar{\Psi} \Gamma_{A A^{\prime}}^{p \uparrow} \Psi \bar{\Psi} \Gamma_{A A^{\prime}}^{p \downarrow} \Psi\right\},
\end{aligned}
$$

with 18 scalar operators

$$
\Gamma_{A A^{\prime}}^{s \uparrow}=\left(\begin{array}{cc}
0 & i \gamma_{5} \tau_{A} \lambda_{A^{\prime}} \\
0 & 0
\end{array}\right), \quad \Gamma_{A A^{\prime}}^{s \downarrow}=\left(\begin{array}{cc}
0 & 0 \\
i \gamma_{5} \tau_{A} \lambda_{A^{\prime}} & 0
\end{array}\right)
$$

and 18 pseudoscalar operators

$$
\Gamma_{A A^{\prime}}^{p \uparrow}=\left(\begin{array}{cc}
0 & \tau_{A} \lambda_{A^{\prime}} \\
0 & 0
\end{array}\right), \quad \Gamma_{A A^{\prime}}^{p \downarrow}=\left(\begin{array}{cc}
0 & 0 \\
\tau_{A} \lambda_{A^{\prime}} & 0
\end{array}\right) .
$$

From these expressions we obtain the scattering kernel

$$
\hat{K}=\Gamma_{i} K_{i j} \bar{\Gamma}_{j}
$$

where $\Gamma_{i}$ are the 36 operators defined above,

$$
\bar{\Gamma}_{i}=\gamma^{0} \Gamma_{i}^{\dagger} \gamma^{0}
$$

and

$$
K_{i j}=4 H \delta_{i j}
$$

Repeated operator indices are summed over, unless stated otherwise.

Vertices describing the coupling of an external source to a bare quark are generalized to Nambu-Gorkov space in the following way:

$$
\hat{\Gamma} \rightarrow(\hat{\Gamma})_{N G} \equiv\left(\begin{array}{cc}
\hat{\Gamma} & 0 \\
0 & -C \hat{\Gamma}^{T} C
\end{array}\right)
$$

This guarantees that quark-antiquark bilinears remain unchanged, $\bar{\Psi}(\hat{\Gamma})_{N G} \Psi=\bar{q} \hat{\Gamma} q$.

\section{CFL ground state}

Before we construct the mesonic excitations, we have to determine the ground state of the system. For equal quark masses the CFL phase can be characterized by the equality of three scalar diquark condensates in the color and flavor antitriplet channel,

$$
s_{22}=s_{55}=s_{77},
$$

where

$$
s_{A A^{\prime}}=\left\langle q^{T} C \gamma_{5} \tau_{A} \lambda_{A^{\prime}} q\right\rangle
$$


In general, these condensates are accompanied by induced color-flavor sextet condensates. These are, however, small and can be neglected. If the $S U(3)$-flavor symmetry is explicitly broken by unequal quark masses, Eq. (11) does no longer hold exactly, but the three condensates may differ from each other.

To obtain the ground state we must minimize the thermodynamic potential (per volume $V$ ),

$$
\Omega\left(T,\left\{\mu_{i}\right\}\right)=-\frac{T}{V} \ln \mathcal{Z}\left(T,\left\{\mu_{i}\right\}\right),
$$

where $\mathcal{Z}\left(T,\left\{\mu_{i}\right\}\right)$ is the grand partition function at temperature $T$ and a given set of chemical potentials $\left\{\mu_{i}\right\}$. For $\beta$ equilibrated matter, these can be expressed in terms of the quark number chemical potential $\mu$, the electric charge chemical potential $\mu_{Q}$, and two color chemical potentials $\mu_{3}$ and $\mu_{8}$ [47].

For the CFL phase in mean-field approximation, $\Omega$ is given by

$$
\begin{aligned}
\Omega\left(T,\left\{\mu_{i}\right\}\right)= & -T \sum_{n} \int \frac{d^{3} p}{(2 \pi)^{3}} \frac{1}{2} \operatorname{Tr} \ln \left(\frac{1}{T} S^{-1}\left(i \omega_{n}, \vec{p}\right)\right) \\
& +\frac{1}{4 H} \sum_{A=2,5,7}\left|\Delta_{A}\right|^{2},
\end{aligned}
$$

where the gap parameters $\Delta_{A}$ are related to the diquark condensates,

$$
\Delta_{A}=-2 H s_{A A} .
$$

The inverse dressed propagator reads

$$
S^{-1}(p)=\left(\begin{array}{cc}
\not p+\hat{\mu} \gamma^{0}-\hat{m} & \sum_{A=2,5,7} \Delta_{A} \gamma_{5} \tau_{A} \lambda_{A} \\
-\sum_{A=2,5,7} \Delta_{A}^{*} \gamma_{5} \tau_{A} \lambda_{A} & \not p-\hat{\mu} \gamma^{0}-\hat{m}
\end{array}\right) .
$$

Here $\hat{\mu}$ denotes the diagonal matrix in color and flavor space which is given by the set of chemical potentials $\left\{\mu_{i}\right\}$.

In order to determine the ground state, $\Omega$ must be minimized with respect to the gap parameters $\Delta_{A}$, leading to three gap equations

$$
\frac{\partial \Omega}{\partial \Delta_{A}^{*}}=0, \quad A=2,5,7 .
$$

Furthermore, we require the solutions to be color and electrically neutral in the presence of leptons. In general, this leads to three additional equations,

$$
n_{i} \equiv-\frac{\partial \Omega_{t o t}}{\partial \mu_{i}}=0, \quad i=Q, 3,8
$$

where $\Omega_{\text {tot }}=\Omega+\Omega_{\text {leptons }}$ is the sum of the quark part, Eq. (14), and the contribution of the leptons. Thus, altogether we have a set of six coupled equations for $\Delta_{A}$ and $\mu_{i}$ which must be solved simultaneously. This has been done many times before, and we can refer to the

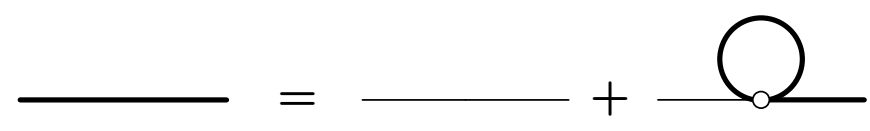

FIG. 1: Dyson equation for the dressed Nambu-Gorkov quark propagator (thick line). The thin line indicates to the bare propagator.

literature for technical details, e.g., Refs. [47-50]. In the present article, we restrict ourselves to the (fully gapped) CFL phase at zero temperature. In this case, color neutral quark matter is electrically neutral without leptons [51] and $\mu_{Q}=0$. Moreover, we consider isospin symmetry, $m_{u}=m_{d}$, so that $\mu_{3}$ vanishes as well.

The gap equations (17) can be derived from the Dyson equation for the dressed quark propagator, too, diagrammatically shown in Fig. 1. This is well known, but some details are useful in our later discussion. Therefore, we present this derivation in Appendix A.

\section{Axial transformations}

In the chiral limit, $m_{u}=m_{d}=m_{s}=0$, the Lagrangian, Eq. (1), is invariant under $S U(3)_{\text {color }} \times U(3)_{L} \times$ $U(3)_{R}$ transformations. In the CFL phase, this symmetry is spontaneously broken to the diagonal vector subgroup, $S U(3)_{\text {color }+V}$. This is reflected by nonzero values for the condensates, Eq. (11). Axial transformations,

$$
q \rightarrow q^{\prime}=\exp \left(i \theta_{a} \gamma_{5} t_{a}\right) q,
$$

then connect a continuous set of degenerate ground states for the CFL phase. Here $t_{a}=\frac{\tau_{a}}{2}, a=0, \ldots, 8$, where $\tau_{1}, \ldots, \tau_{8}$ denote the eight Gell-Mann matrices in flavor space, as before, and $\tau_{0}=\sqrt{2 / 3} \mathbb{1}_{f}$. These transformations can be parameterized by nine pseudoscalar Goldstone bosons. In order to identify the operators corresponding to Goldstone boson excitations, we inspect the effect of an axial transformation with specified quantum numbers on the condensates, Eq. (11). In Nambu-Gorkov formalism this can be described as follows

$$
\left\langle\bar{\Psi} \Gamma_{i} \Psi\right\rangle \rightarrow\left\langle\bar{\Psi}^{\prime} \Gamma_{i} \Psi^{\prime}\right\rangle \equiv\left\langle\bar{\Psi} \Gamma_{i}^{\prime} \Psi\right\rangle,
$$

for $\Gamma_{i}=\Gamma_{A A}^{s \uparrow}$ and $\Gamma_{A A}^{s \downarrow}$. Considering infinitesimal transformations, we find

$$
\Gamma_{i}^{\prime}=\Gamma_{i}+i \theta_{a} \delta \Gamma_{i, a},
$$

with

$$
\delta \Gamma_{i, a}=\left\{\left(\gamma_{5} t_{a}\right)_{N G}, \Gamma_{i}\right\},
$$

where $\{A, B\}=A B+B A$ denotes the anticommutator. 


$\left.\begin{array}{ccc}\text { "meson" } & t_{j} & \Gamma_{j} \\ \hline \pi^{+} & \frac{\tau_{1}+i \tau_{2}}{2 \sqrt{2}} & \Gamma_{57}^{p \uparrow}, \Gamma_{75}^{p \downarrow} \\ \pi^{-} & \frac{\tau_{1}-i \tau_{2}}{2 \sqrt{2}} & \Gamma_{75}^{p \uparrow}, \Gamma_{57}^{p \downarrow} \\ K^{+} & \frac{\tau_{4}+i \tau_{5}}{2 \sqrt{2}} & \Gamma_{27}^{p \uparrow}, \Gamma_{72}^{p \downarrow} \\ K^{-} & \frac{\tau_{4}-i \tau_{5}}{2 \sqrt{2}} & \Gamma_{72}^{p \uparrow}, \Gamma_{27}^{p \downarrow} \\ K^{0} & \frac{\tau_{6}+i \tau_{7}}{2 \sqrt{2}} & \Gamma_{25}^{p \uparrow}, \Gamma_{52}^{p \downarrow} \\ \bar{K}^{0} & \frac{\tau_{6}-i \tau_{7}}{2 \sqrt{2}} & \Gamma_{52}^{p \uparrow}, \Gamma_{25}^{p \downarrow} \\ \pi^{0} & \frac{\tau_{3}}{2} & \Gamma_{55}^{p \uparrow}, \Gamma_{77}^{p \uparrow}, \Gamma_{55}^{p \downarrow}, \Gamma_{77}^{p \downarrow} \\ \eta_{8} & \frac{\tau_{8}}{2} \\ \eta_{0} & \frac{\tau_{0}}{2}\end{array}\right\} \quad\left\{\begin{array}{c}\Gamma_{22}^{p \uparrow}, \Gamma_{55}^{p \uparrow}, \Gamma_{77}^{p \uparrow}, \\ \Gamma_{22}^{p \downarrow}, \Gamma_{55}^{p \downarrow}, \Gamma_{77}^{p \downarrow}\end{array}\right\}$

TABLE I: Pseudoscalar Goldstone modes, corresponding flavor operators $t_{j}$, and Nambu-Gorkov operators $\Gamma_{j}$ contributing to the quark-meson vertex. The $\Gamma_{j}$ are obtained by evaluating Eq. (22) for $t_{a}=t_{j}$ and all possible $\Gamma_{i}=\Gamma_{A A}^{s \uparrow}$ or $\Gamma_{A A}^{s \downarrow}$.

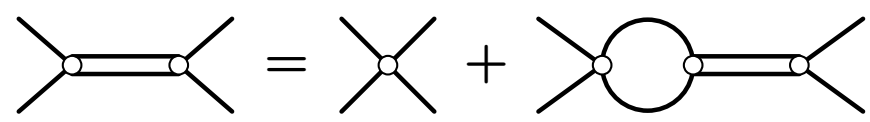

FIG. 2: RPA equation for the T-matrix in Nambu-Gorkov space.

The Goldstone bosons are coupled to the quarks by vertices related to $\delta \Gamma_{i, a}$ (see Ref. [32] for a detailed discussion). Thus, by evaluating Eq. (22) for $\Gamma_{i}=\Gamma_{A A}^{s \uparrow}$ and $\Gamma_{A A}^{s \downarrow}$, we can determine the operators which contribute to the vertex of a given Goldstone boson. It turns out that the assignment is most simple if we work in the "particle basis" instead of using hermitian flavor operators. In this case all flavored mesons are coupled to only two Nambu-Gorkov operators, while the hidden-flavor mesons are coupled to four $\left(\pi^{0}\right)$ or six $\left(\eta_{8}, \eta_{0}\right)$ operators. The results are summarized in Table I. As we will see below, this assignment remains basically unchanged when we include finite quark masses.

\section{E. Mesonic excitations}

Iterating the scattering kernel as illustrated in Fig. 2 leads to the RPA equation for the T-matrix in NambuGorkov space,

$$
\hat{T}=\hat{K}+\hat{K} \hat{J} \hat{T}
$$

Since the interaction is separable, this operator equation can be converted into a matrix equation by using Eq. (7) for the scattering kernel $\hat{K}$, an analogous expression for $\hat{T}$,

$$
\hat{T}=\Gamma_{i} T_{i j} \bar{\Gamma}_{j}
$$

and defining

$$
\bar{\Gamma}_{i} \hat{J} \Gamma_{j}=J_{i j}
$$

One finds

$$
T=K+K J T
$$

with the solution

$$
T(q)=(\mathbb{1}-K J(q))^{-1} K=\left(\frac{1}{4 H}-J(q)\right)^{-1},
$$

where the last equality follows from Eq. (9). The polarization matrix, corresponding to the loop in Fig. 2, is given by

$$
J_{i j}(q)=i \int \frac{d^{4} k}{(2 \pi)^{4}} \frac{1}{2} \operatorname{Tr}\left[\bar{\Gamma}_{i} S(k+q) \Gamma_{j} S(k)\right] .
$$

Here we have introduced a "vacuum-like" notation for brevity. In medium we should replace

$$
q \rightarrow\left(\begin{array}{c}
i \omega_{m} \\
\vec{q}
\end{array}\right), \quad k \rightarrow\left(\begin{array}{c}
i \omega_{n} \\
\vec{k}
\end{array}\right)
$$

and

$$
i \int \frac{d^{4} k}{(2 \pi)^{4}} \rightarrow-T \sum_{n} \int \frac{d^{3} k}{(2 \pi)^{3}}
$$

with bosonic Matsubara frequencies $i \omega_{m}$ and fermionic Matsubara frequencies $i \omega_{n}$. In the end, the result should be analytically continued to real external energies. We will use this notation throughout this paper. For our numerical results, we will introduce a 3-momentum cutoff $\Lambda$ to regularize the divergent integrals.

The matrices $T$ and $J$ are $36 \times 36$ matrices in operator space, corresponding to the 36 operators defined in Eqs. (5) and (6). It turns out, however, that $J$ and, thus, $T$ are block diagonal, i.e., only certain combinations of operators occur. More precisely, scalar operators do not mix with pseudoscalar ones, and each of the resulting $18 \times 18$ blocks can be decomposed further into six $2 \times 2$ blocks and one $6 \times 6$ block. These blocks carry different quantum numbers and reflect the assignment to different meson modes, as given in Table $\mathrm{I}^{2}$ In particular, the $2 \times 2$ blocks correspond to the flavored mesons. The $6 \times 6$ blocks, on the other hand, describe the hidden-flavor mesons, i.e., $\pi^{0}, \eta_{8}$ and $\eta_{0}$ in the pseudoscalar case. As in vacuum, these states are mixed for unequal quark masses.

This assignment can be tested by coupling the Tmatrix to an external meson source, as illustrated in Fig. 3. To that end, we evaluate the loop integral

\footnotetext{
${ }^{2}$ In Table I we have listed the pseudoscalar mesons only, but the scalar sector is completely analogous.
} 


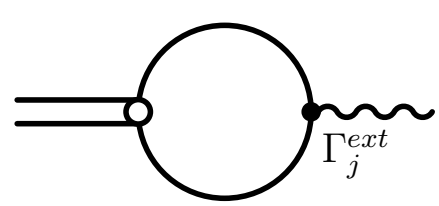

FIG. 3: Coupling the T-matrix (double line) to an external meson source (wavy line).

$$
I_{i j}(q)=i \int \frac{d^{4} k}{(2 \pi)^{4}} \frac{1}{2} \operatorname{Tr}\left[\bar{\Gamma}_{i} S(k+q)\left(\Gamma_{j}^{e x t}\right)_{N G} S(k)\right]
$$

where $\Gamma_{j}^{e x t}$ denotes the vertex of the external source to the quark. It is given by $\Gamma_{j}^{e x t}=t_{j}$ for scalar sources and $\Gamma_{j}^{e x t}=i \gamma_{5} t_{j}$ for pseudoscalar sources. Here $t_{j}$ is one of the generators in flavor space listed in Table I, and $\left(\Gamma_{j}^{e x t}\right)_{N G}$ is the generalization of this vertex to NambuGorkov space, as defined in Eq. (10).

The blocks can be diagonalized by unitary transformations,

$$
J^{\prime}=W J W^{\dagger},
$$

with $W$ being an (in general 4-momentum dependent) unitary matrix and

$$
J_{i j}^{\prime}=J^{(i)} \delta_{i j} .
$$

Defining new operators

$$
\Gamma_{j}^{\prime}=\Gamma_{i} W_{i j}^{\dagger}
$$

$J^{\prime}$ can be rewritten as

$$
J_{i j}^{\prime}(q)=i \int \frac{d^{4} k}{(2 \pi)^{4}} \frac{1}{2} \operatorname{Tr}\left[\bar{\Gamma}_{i}^{\prime} S(k+q) \Gamma_{j}^{\prime} S(k)\right] .
$$

Since the scattering kernel remains diagonal in this new basis,

$$
\hat{K}=4 H \Gamma_{i} \bar{\Gamma}_{i}=4 H \Gamma_{i}^{\prime} \bar{\Gamma}_{i}^{\prime},
$$

the T-matrix becomes diagonal as well

$$
\hat{T}=\Gamma_{i}^{\prime} T^{(i)} \bar{\Gamma}_{i}^{\prime}
$$

with

$$
T^{(i)}(q)=\frac{1}{\frac{1}{4 H}-J^{(i)}(q)} .
$$

In the vicinity of a pole, we can parameterize these modes like a free boson with mass $m_{i}$ in the presence of a chemical potential $\mu_{i}$ corresponding to this particular boson,

$$
T^{(i)}(q) \approx \frac{-g_{i}^{2}}{\left(q_{0}+\mu_{i}\right)^{2}-c_{i}^{2} \vec{q}^{2}-m_{i}^{2}} .
$$

Here $g_{i}$ can be interpreted as a coupling constant of the boson to an external quark, and $c_{i}$ denotes the in-medium group velocity. In this article we restrict ourselves to $\vec{q}=$ 0 in order to keep artifacts of the 3-momentum cutoff as small as possible.

The various modes $T^{(i)}$ describe bosonic excitations of the CFL ground state. Because of the formal quarkantiquark structure of the polarization loops, we will call these excitations "mesons". However, it should be kept in mind that the propagators and vertices entering the polarization loops live in Nambu-Gorkov space and therefore in principle describe quark-antiquark as well as diquark and antidiquark (or di-hole) excitations. In vacuum or in a normal-conducting medium, these are independent modes, protected by the conserved baryon number. In the CFL phase, however, baryon number is broken, and quark-antiquark, diquark, and antidiquark states can mix. As our model Lagrangian does not contain quarkantiquark interactions, our "mesons" are in fact superpositions of diquarks and antidiquarks or, more important, di-holes.

In vacuum we have nine scalar and nine pseudoscalar diquarks and nine scalar and nine pseudoscalar antidiquarks. Since the total number of states does not change when the states are mixed, there must be 36 meson states in the CFL phase, 18 scalars and 18 pseudoscalars. (If we included quark-antiquark interactions in our model, we would obtain 27 scalars and 27 pseudoscalars.) As we will see below, nine pseudoscalars are massless in the chiral limit, while the others stay heavy. This has been found in Ref. [44], too, within a similar model.

\section{F. Pseudoscalar meson decay constants}

As in vacuum, the pseudoscalar mesons can decay weakly. The decay amplitudes are related to the loop integrals

$F_{i j}^{\prime \mu}(q)=i \int \frac{d^{4} k}{(2 \pi)^{4}} \frac{1}{2} \operatorname{Tr}\left[\bar{\Gamma}_{i}^{\prime} S(k+q)\left(\gamma^{\mu} \gamma_{5} t_{j}\right)_{N G} S(k)\right]$,

describing the coupling of the meson $i$ (i.e., the one which corresponds to the operator $\left.\Gamma_{i}^{\prime}\right)$ to an external axial current $A_{5 j}^{\mu}{ }^{3}$ In the following, we are mostly interested in flavored mesons, which are the main focus of our studies. Each flavored meson $i$ couples to only one $t_{j}$, as listed in Table I. ${ }^{4}$ For simplicity, we denote the flavor operator which fits to the meson $i$ by $t_{i}$.

\footnotetext{
${ }^{3}$ Strictly speaking, Eq. (40) describes the time-reversed process, i.e., the production of a meson by an incoming axial current. This choice was made for later convenience in Sec. III B. There we apply chiral Ward identities, which are formulated for incoming axial currents, see Eq. (52).

${ }^{4}$ Note that the opposite is not true: Since we have 18 pseudoscalars, there are in general two meson states $i$ which couple to a given flavor operator $t_{j}$.
} 
The meson decay constants are related to the on-shell values of these amplitudes,

$$
f_{i} q^{\mu}=\left.i g_{i} F_{i i}^{\prime \mu}(q)\right|_{\text {on-shell }},
$$

with no summation over the index $i$ on the r.h.s. The coupling constant $g_{i}$ has been defined implicitly in Eq. (39).

In general, there are different values for the time-like $(\mu=0)$ and the space-like $(\mu=1,2,3)$ decay constants, which differ by the group velocity of the Goldstone modes. However, since we only consider mesons with vanishing 3-momenta in this article, we are restricted to the time-like decay constants.

\section{EQUAL QUARK MASSES}

It is rather instructive to investigate the simplified case of equal quark masses, $m_{u}=m_{d}=m_{s} \equiv m$. In this case, we have only one gap parameter $\Delta_{2}=\Delta_{5}=\Delta_{7} \equiv \Delta$ and one common chemical potential $\mu$ in electrically and color neutral CFL matter. Moreover, the set of the 18 pseudoscalar meson states consists of two $S U(3)$ octets and two singlets, with all mesons in the same multiplet being degenerate. ${ }^{5}$ Finally, there is no stress caused by quark mass or chemical potential differences, which could act as an effective meson chemical potential as in Eq. (39). Hence, for vanishing 3-momenta, the pole approximation for the T-matrix, Eq. (39), becomes

$$
T^{(i)}\left(q_{0}\right) \approx \frac{-g_{i}^{2}}{q_{0}^{2}-m_{i}^{2}}
$$

This yields for the decay constants

$$
f_{i} q_{0}=\left.i g_{i} F_{i i}^{\prime 0}(q)\right|_{q_{0}=m_{i}, \vec{q}=0} .
$$

We will often refer to the states of the lowest pseudoscalar octet as "pions" and denote their masses, couplings, and decay constants by $m_{\pi}, g_{\pi}$, and $f_{\pi}$, respectively.

\section{A. Dressed vertex functions}

Attaching an external axial current to both sides of the Dyson equation for the dressed quark propagator (see upper line of Fig. 4), we obtain a selfconsistency equation for the dressed axial vertex,

$$
\begin{aligned}
& \Gamma_{5 j}^{\mu}(p ; q)=\left(\gamma^{\mu} \gamma_{5} t_{j}\right)_{N G} \\
& +4 i H \Gamma_{i} \int \frac{d^{4} k}{(2 \pi)^{4}} \frac{1}{2} \operatorname{Tr}\left[\bar{\Gamma}_{i} S(k+q) \Gamma_{5 j}^{\mu}(k ; q) S(k)\right] .
\end{aligned}
$$

\footnotetext{
5 The degeneracy in the octets is due to the residual $S U(3)_{\text {color }+V}$ symmetry of the CFL phase. Thus, even though we start from a $U(3)$ invariant Lagrangian in our model, there is no unbroken symmetry which relates the singlet states to the octets.
}

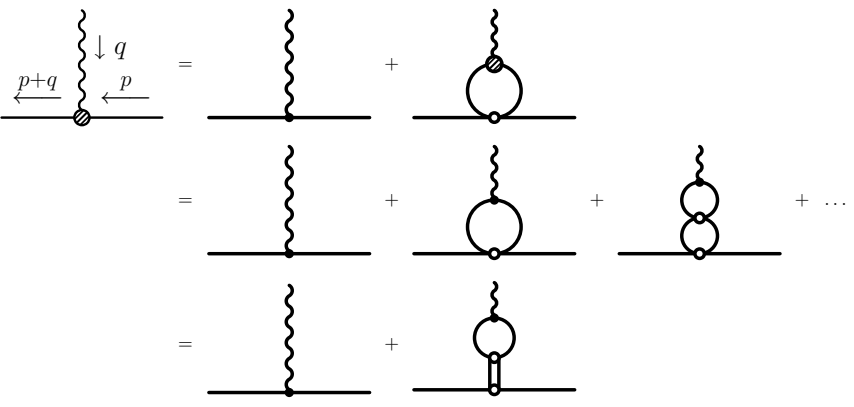

FIG. 4: Vertex function for an external axial current (wavy line) coupled to a quark. The shaded circles (black dots) indicate dressed (bare) vertices. The open circles correspond to quark-quark vertices and the double line to the T-matrix, as in Fig. 2.

The first term on the r.h.s. corresponds to the bare vertex, while the second term contains the dressed vertex again. Thus, iterating the equation, the dressed vertex can be written as a Born series of quark-antiquark loops (second line of Fig. 4), with the bare vertex attached to the last loop. Employing the quark-antiquark T-matrix, this could be rewritten (last line of Fig. 4) as

$$
\Gamma_{5 j}^{\mu}(p ; q)=\left(\gamma^{\mu} \gamma_{5} t_{j}\right)_{N G}+T^{(i)}(q) \Gamma_{i}^{\prime} F_{i j}^{\prime \mu}(q),
$$

with $F_{i j}^{\prime \mu}$ as defined in Eq. (40). We have seen earlier that a given flavor operator $t_{j}$ couples to only two meson modes $i$ in flavored channels. We may call these modes " $\pi_{j}$ " and " $\pi_{j}^{\prime}$ ". Then, for $\vec{q}=0$ and $q_{0}$ approaching $m_{\pi}$, we obtain

$\Gamma_{5 j}^{0}\left(p ; q_{0} \rightarrow m_{\pi}\right)=i \frac{g_{\pi} f_{\pi} q_{0}}{q_{0}^{2}-m_{\pi}^{2}} \Gamma_{\pi_{j}}^{\prime}+$ non-singular terms.

Similarly, we have an equation for the dressed pseudoscalar vertex

$$
\begin{aligned}
& \Gamma_{5 j}(p ; q)=\left(i \gamma_{5} t_{j}\right)_{N G} \\
& +4 i H \Gamma_{i} \int \frac{d^{4} k}{(2 \pi)^{4}} \frac{1}{2} \operatorname{Tr}\left[\bar{\Gamma}_{i} S(k+q) \Gamma_{5 j}(k ; q) S(k)\right],
\end{aligned}
$$

which could be rewritten as

$$
\Gamma_{5 j}(p ; q)=\left(i \gamma_{5} t_{j}\right)_{N G}+T^{(i)}(q) \Gamma_{i}^{\prime} I_{i j}^{\prime}(q),
$$

with

$$
I_{i j}^{\prime}(q)=i \int \frac{d^{4} k}{(2 \pi)^{4}} \frac{1}{2} \operatorname{Tr}\left[\bar{\Gamma}_{i}^{\prime} S(k+q)\left(i \gamma_{5} t_{j}\right)_{N G} S(k)\right] .
$$

Thus, approaching the pole, we find

$\Gamma_{5 j}\left(p ; q_{0} \rightarrow m_{\pi}\right)=-\frac{g_{\pi}^{2} I^{(\pi)}}{q_{0}^{2}-m_{\pi}^{2}} \Gamma_{\pi_{j}}^{\prime}+$ non-singular terms,

with

$$
I^{(\pi)}=I_{\pi \pi}^{\prime}\left(q_{0}^{2}=m_{\pi}^{2}\right)
$$




\section{B. Chiral Ward-Takahashi identity}

It can be shown on general grounds that the exact vertex functions and inverse propagators must satisfy the following axial Ward-Takahashi identity:

$$
\begin{aligned}
& q_{\mu} \Gamma_{5 j}^{\mu}(p ; q)+2 m i \Gamma_{5, j}(p ; q) \\
= & S^{-1}(p+q)\left(\gamma_{5} t_{j}\right)_{N G}+\left(\gamma_{5} t_{j}\right)_{N G} S^{-1}(p) .
\end{aligned}
$$

In Appendix B we show by using the gap equation that the vertex functions defined above and the inverse propagator (16) are consistent with this relation.

We now evaluate this equation for $\vec{q}=0$ and $q_{0} \rightarrow$ $m_{\pi}$. In the chiral limit, $m=0$, only the axial vertex contributes, and we find from Eq. (46) that the l.h.s. is given by

$$
q_{\mu} \Gamma_{5 j}^{\mu}=i \frac{q_{0}^{2}}{q_{0}^{2}-m_{\pi}^{2}} g_{\pi} f_{\pi} \Gamma_{\pi_{j}}^{\prime}+\text { non-singular terms. }
$$

In general, this has a singularity at $q_{0}=m_{\pi}$. On the other hand, one can easily see that the r.h.s. of Eq. (52) remains finite. We thus conclude that the singularity on the l.h.s. must be suppressed, i.e., either $m_{\pi}=0$ or $g_{\pi} f_{\pi}=0$. In fact, from the symmetry breaking pattern we expect nine pseudoscalar Goldstone bosons. Therefore, both scenarios should be realized, i.e., nine pseudoscalar mesons (one octet and one singlet) are massless and the others are massive.

For the massless solution, we can now evaluate Eq. (52) directly at $q=0$. This yields

$$
i g_{\pi} f_{\pi} \Gamma_{\pi_{j}}^{\prime(0)}=\left\{\left(\gamma_{5} t_{j}\right)_{N G}, S^{-1}(p)\right\} .
$$

Inserting Eq. (16) for the inverse propagator with $m=0$ and taking the freedom to choose the gap parameter $\Delta$ to be real, we find the solution

$$
g_{\pi} f_{\pi}=\Delta
$$

with the assignment (cf. Eq. (22))

$$
\Gamma_{\pi_{j}}^{\prime(0)}=-\sum_{A=2,5,7}\left\{\left(\gamma_{5} t_{j}\right)_{N G},\left(\Gamma_{A A}^{s \uparrow}-\Gamma_{A A}^{s \downarrow}\right)\right\} .
$$

Eq. (55) may be viewed as a generalization of the wellknown Goldberger-Treiman relation in vacuum [32].

Explicit evaluation of Eq. (56) yields

$$
\begin{array}{lll}
\Gamma_{\pi^{+}}^{\prime(0)}=\frac{-i}{\sqrt{2}}\left(\Gamma_{57}^{p \uparrow}-\Gamma_{75}^{p \downarrow}\right), & \Gamma_{\pi^{-}}^{\prime(0)}=\frac{-i}{\sqrt{2}}\left(\Gamma_{75}^{p \uparrow}-\Gamma_{57}^{p \downarrow}\right), \\
\Gamma_{K^{+}}^{\prime(0)}=\frac{i}{\sqrt{2}}\left(\Gamma_{27}^{p \uparrow}-\Gamma_{72}^{p \downarrow}\right), & \Gamma_{K^{-}}^{\prime(0)}=\frac{i}{\sqrt{2}}\left(\Gamma_{72}^{p \uparrow}-\Gamma_{27}^{p \downarrow}\right), \\
\Gamma_{K^{0}}^{\prime(0)}=\frac{-i}{\sqrt{2}}\left(\Gamma_{25}^{p \uparrow}-\Gamma_{52}^{p \downarrow}\right), & \Gamma_{\bar{K}^{0}}^{\prime(0)}=\frac{-i}{\sqrt{2}}\left(\Gamma_{52}^{p \uparrow}-\Gamma_{25}^{p \downarrow}\right),
\end{array}
$$

for the flavored mesons, and

$$
\begin{aligned}
& \Gamma_{\pi^{0}}^{\prime(0)}=-\frac{i}{2}\left(\Gamma_{55}^{p \uparrow}-\Gamma_{77}^{p \uparrow}-\Gamma_{55}^{p \downarrow}+\Gamma_{77}^{p \downarrow}\right), \\
& \Gamma_{\eta_{8}}^{\prime(0)}=\frac{-i}{2 \sqrt{3}}\left(2 \Gamma_{22}^{p \uparrow}-\Gamma_{55}^{p \uparrow}-\Gamma_{77}^{p \uparrow}-2 \Gamma_{22}^{p \downarrow}+\Gamma_{55}^{p \downarrow}+\Gamma_{77}^{p \downarrow}\right), \\
& \Gamma_{\eta_{0}}^{\prime(0)}=-i \sqrt{\frac{2}{3}}\left(\Gamma_{22}^{p \uparrow}+\Gamma_{55}^{p \uparrow}+\Gamma_{77}^{p \uparrow}-\Gamma_{22}^{p \downarrow}-\Gamma_{55}^{p \downarrow}-\Gamma_{77}^{p \downarrow}\right),
\end{aligned}
$$

for the mesons with hidden flavor. Thus, antiparticle modes are related to each other as

$$
\Gamma_{\bar{\pi}_{j}}^{\prime(0)}=\bar{\Gamma}_{\pi_{j}}^{\prime(0)},
$$

(with $\pi^{0}, \eta_{8}$ and $\eta_{0}$ being their own antiparticles) as it should be.

The results above are consistent with the operators identified in Ref. [32] following a similar reasoning. Except for an arbitrary phase, Eqs. (57) and (58) also agree with the result of diagonalizing the meson polarization function $J_{i j}$ for vanishing quark masses (see Sect. IIE). We should keep in mind, however, that we have evaluated the meson vertices at the pole only. In general, the diagonalization of the T-matrix leads to 4-momentum dependent vertex functions with 4-momentum dependent weights for the contributing operators. For instance, the $\pi^{+}$vertex can be written as

$$
\Gamma_{\pi^{+}}^{\prime}(q)=-i\left(\sin \varphi(q) \Gamma_{57}^{p \uparrow}-\cos \varphi(q) \Gamma_{75}^{p \downarrow}\right),
$$

with some function $\varphi(q)$ and an arbitrary overall phase which we have chosen to be purely imaginary to comply with Eq. (57). For equal quark masses, $\varphi(0)=\frac{\pi}{4}$ and we recover $\Gamma_{\pi_{j}}^{\prime}(0)=\Gamma_{\pi_{j}}^{\prime(0)}$, Eq. (57).

Next, we evaluate Eq. (52) for non-vanishing (but still equal) quark masses $m$. In this case the pseudoscalar vertex contributes a pole at $\vec{q}=0$ and $q_{0} \rightarrow m_{\pi}$, which is present for any choice of $m_{\pi}$ (see Eq. (50)). Since the r.h.s. of Eq. (52) remains non-singular, this pole must be cancelled by the pole in the axial part (unless $g_{\pi}^{2} I^{(\pi)}$ vanishes). This means that the formerly massless mesons receive a mass which is determined by the requirement that the residues of the axial and the pseudoscalar pole cancel each other. One finds

$$
m_{\pi}^{2} f_{\pi}^{2}=2 m g_{\pi} f_{\pi} I^{(\pi)} .
$$

This is an exact relation, valid for arbitrary values of $m$. We can now perform a chiral expansion of this equation to leading order. This amounts to replace $g_{\pi} f_{\pi}$ by $\Delta$ and to evaluate

$$
\begin{aligned}
I^{(\pi)}= & i \int \frac{d^{4} k}{(2 \pi)^{4}} \\
& \left.\quad \frac{1}{2} \operatorname{Tr}\left[\bar{\Gamma}_{\pi}^{\prime}(q) S(k+q)\left(i \gamma_{5} t_{\pi}\right)_{N G} S(k)\right]\right|_{q_{0}=m_{\pi}, \vec{q}=0}
\end{aligned}
$$


to leading nontrivial order in the quark mass. It turns out that $I^{(\pi)}$ vanishes in the chiral limit and the leading order is linear in $m{ }^{6}$ Note that, in principle, $I^{(\pi)}$ depends explicitly (via the quark propagators) and implicitly (via $m_{\pi}$ ) on $m$. One can show, however, that the implicit contributions vanish in leading order. It is therefore consistent to evaluate the integral at $q_{0}=0$, and we obtain

$$
m_{\pi}^{2} f_{\pi}^{2}=8 A m^{2}+\text { higher orders }
$$

with

$$
\begin{aligned}
A=\frac{\Delta}{4} i \int & \frac{d^{4} k}{(2 \pi)^{4}} \\
& \left.\left(\frac{d}{d m} \frac{1}{2} \operatorname{Tr}\left[\bar{\Gamma}_{\pi}^{\prime(0)} S(k)\left(i \gamma_{5} t_{\pi}\right)_{N G} S(k)\right]\right)\right|_{m=0}
\end{aligned}
$$

and $\Gamma_{\pi}^{\prime(0)} \equiv \Gamma_{\pi}^{\prime}(0)$ as given in Eq. (57). This expression can be evaluated exactly. The result is given in Eq. (E1) in the appendix. Expanding that formula in $\Delta$, we find

$$
A=\frac{\Delta^{2}}{8 \pi^{2}}\left(-\ln y^{2}-2+\frac{4}{3} \ln 2+\ln \left(x^{2}-1\right)\right)+\ldots,
$$

where we have introduced the abbreviations

$$
x=\frac{\Lambda}{\mu}, \quad y=\frac{\Delta}{\mu},
$$

with $\Lambda$ being the 3 -momentum cutoff. This should be compared with the weak-coupling result [23],

$$
A_{w c}=\frac{3 \Delta^{2}}{4 \pi^{2}}
$$

An important difference is the logarithmic term in Eq. (65), which does not exist in Eq. (67). In fact, in the beginning, there was a controversy about the correct weak-coupling limit, and similar logarithmic terms have also been discussed in the literature [26, 30, 32-34, 52]. To be precise, it was found that the leading contribution to $A$ in weak coupling is given by [30]

$$
A_{\Delta \bar{\Delta}}=-\frac{\Delta \bar{\Delta}}{8 \pi^{2}} \ln y^{2}
$$

where $\bar{\Delta}$ is the antiparticle gap. In the NJL model, the gap function is energy independent and, hence, $\bar{\Delta}=$ $\Delta$. Therefore, our result, Eq. (65), is consistent with Eq. (68). Moreover, if we introduce particle and antiparticle gaps by hand as independent constants in the quark propagator, we recover Eq. (68) in leading order.

\footnotetext{
6 If we had taken into account dynamical quark masses it would be linear in the constituent quark mass $M$. The leading term on the r.h.s. of Eq. (63) would then $\operatorname{read} 8 A m M$.
}

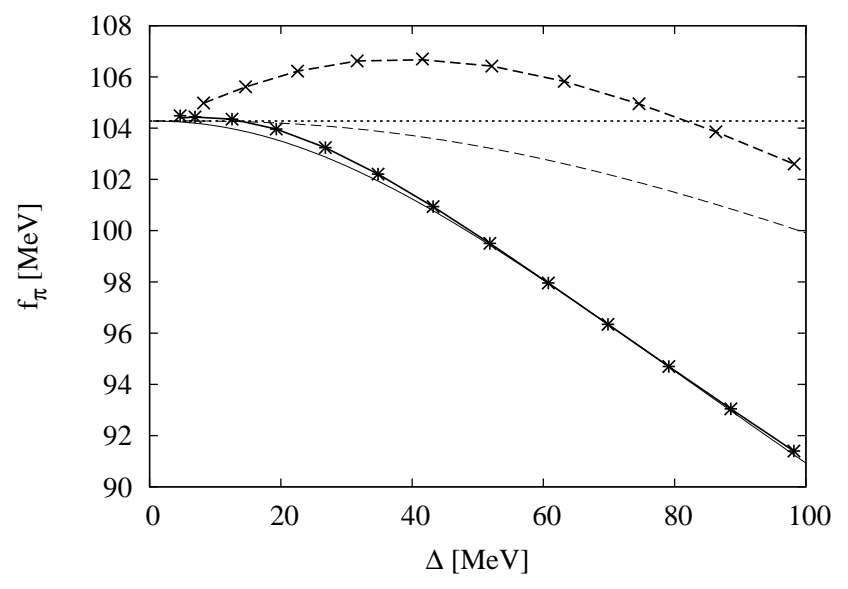

FIG. 5: Pion decay constant $f_{\pi}$ in the chiral limit as a function of the gap parameter $\Delta$ : numerical results (points) in comparison with the semi-analytical formula Eq. (70), neglecting (thin lines) and including (thick lines) the momentum dependence of the vertex function. Asterisks and solid lines: $\Lambda=600 \mathrm{MeV}$; crosses and dashed lines: $\Lambda=700 \mathrm{MeV}$; dotted line: weak-coupling limit, Eq. (69).

On the other hand, as argued in the erratum of Ref. [23] and confirmed in Ref. [53], in a gauge invariant treatment of QCD at weak coupling, the antiparticle gap contributions are cancelled by other terms, and the logarithmic terms drop out. This finally leads to Eq. (67). Of course, gauge invariance is not an issue in the NJL model. Nevertheless, the logarithmic behavior at very weak coupling should be viewed as a model artifact.

\section{NUMERICAL RESULTS}

In the following we present our results for $T=0$ and a fixed quark chemical potential $\mu=500 \mathrm{MeV}$.

\section{A. Pion decay constant in the chiral limit}

We begin with a discussion of the pion decay constant in the chiral limit, i.e., for vanishing quark masses. In Fig. 5 our results are displayed as functions of the gap parameter $\Delta$ for two different values of the cutoff $\Lambda$. The points correspond to the numerical results for $\Lambda=600 \mathrm{MeV}$ (asterisks) and $\Lambda=700 \mathrm{MeV}$ (crosses). To be precise, these calculations have been performed with $m_{u}=m_{d}=m_{s}=0.1 \mathrm{MeV}$ for technical reasons.

The weak-coupling limit, $\Delta \rightarrow 0$, of $f_{\pi}$ has been derived in Ref. [23] from an effective theory involving only fermionic modes in the vicinity of the Fermi surface. The result,

$$
f_{\pi}^{2} \rightarrow \frac{21-8 \ln 2}{18} \frac{\mu^{2}}{2 \pi^{2}}
$$


is universal and should hold in any model exhibiting the same symmetry pattern. ${ }^{7}$ Indeed, our results converge to this limit for $\Delta \rightarrow 0$. In the general case we find, however, deviations from Eq. (69), e.g., about $10 \%$ for $\Lambda=600 \mathrm{MeV}$ and $\Delta \approx 80 \mathrm{MeV}$. Moreover, these deviations depend rather strongly on the cutoff.

In order to understand this behavior and to confirm the correct weak-coupling limit, we employ the semianalytical formula derived in App. D,

$$
f_{\pi}^{2}=\tilde{f}_{\pi}^{2}+\delta f_{\pi}^{2} .
$$

$\tilde{f}_{\pi}^{2}$ thereby describes the contribution to $f_{\pi}$ arising for a constant pion vertex function, i.e., neglecting the energy dependence of the mixing angle $\varphi$ in Eq. (60). This part is given in a closed analytical form in Eq. (D5). $\delta f_{\pi}^{2}$, on the other hand, incorporates the effect of the energy dependence of the vertex function and is given in Eq. (D11). It is proportional to the derivative $d \varphi / d q_{0}$, which is evaluated numerically.

Expanding $\tilde{f}_{\pi}^{2}$ and the analytical factor of $\delta f_{\pi}^{2}$ for small values of $\Delta$ yields

$$
\tilde{f}_{\pi}^{2}=\frac{\mu^{2}}{36 \pi^{2}}\left\{(21-8 \ln 2)-9 y^{2} \ln y^{2}-c_{2} y^{2}+\ldots\right\}
$$

and

$$
\delta f_{\pi}^{2}=\left.\frac{\mu^{2}}{36 \pi^{2}}\left\{-36 y^{2} \ln y^{2}-d_{2} y^{2}+\ldots\right\} \mu \frac{d \varphi}{d q_{0}}\right|_{q=0},
$$

where

$$
\begin{aligned}
& c_{2}=\frac{81}{4}-18 \ln 2-9 \ln \left(x^{2}-1\right)+\frac{45 x^{2}-27}{\left(x^{2}-1\right)^{2}}, \\
& d_{2}=102-56 \ln 2-36 \ln \left(x^{2}-1\right)+\frac{36}{x^{2}-1},
\end{aligned}
$$

and $x$ and $y$ are defined in Eq. (66).

We see that the weak-coupling limit, Eq. (69), is correctly reproduced by the leading term in $\tilde{f}_{\pi}^{2}$, while $\delta f_{\pi}^{2}$ does not contribute to this order, provided $d \varphi / d q_{0}$ does not diverge as strongly as $\left(y^{2} \ln y^{2}\right)^{-1}$ for $\Delta \rightarrow 0$. We therefore expect that $\tilde{f}_{\pi}^{2}$ gives the main contribution to $f_{\pi}$.

As already mentioned, the weak-coupling limit is universal and therefore must be cutoff independent. In the detailed calculations, this results from the fact that in the limit $\Delta \rightarrow 0$ the integrand of the 3-momentum integral in Eq. (D3) becomes proportional to $\delta$-functions at the Fermi surface. While the $y^{2} \ln y^{2}$-term in Eq. (71) is cutoff independent as well, the quadratic term is not universal and dependends on $\Lambda$ via the variable $x$. This

\footnotetext{
7 Strictly speaking, the chemical potential $\mu$ should be replaced by the Fermi momentum $p_{F}$ because in general the Fermi velocity can differ from the speed of light.
}

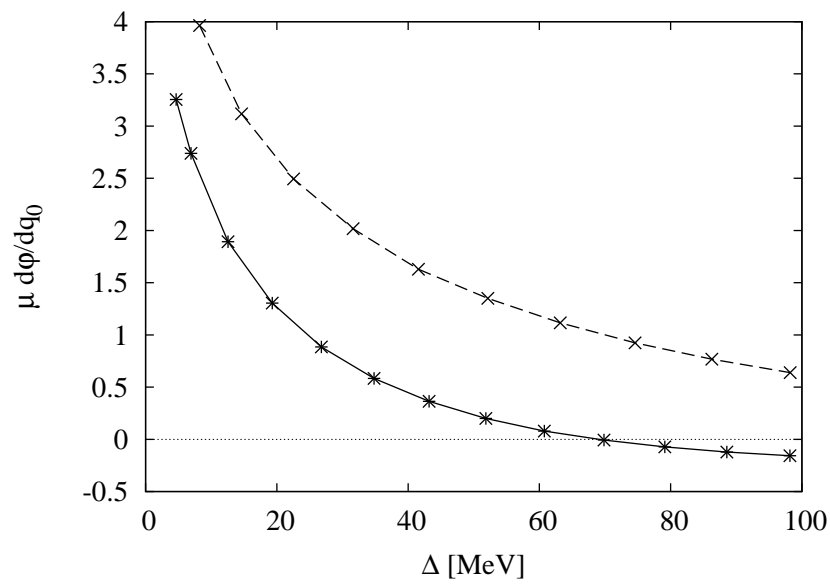

FIG. 6: Derivative of the mixing angle $\varphi$ as a function of the gap parameter $\Delta$. Solid: $\Lambda=600 \mathrm{MeV}$, dashed: $\Lambda=700 \mathrm{MeV}$.

is also the case for the quadratic term in Eq. (72). Anyway, the situation for $\delta f_{\pi}^{2}$ is more complicated because the derivative $d \varphi / d q_{0}$ depends on the cutoff as well.

The results of the semi-analytical formula for $f_{\pi}$ are indicated by the thick lines in Fig. 5. The solid line corresponds to $\Lambda=600 \mathrm{MeV}$ and the dashed line to $\Lambda=700 \mathrm{MeV}$. We have employed the exact formulas, Eqs. (D5) and (D11), for $\tilde{f}_{\pi}^{2}$ and $\delta f_{\pi}^{2}$, respectively, with $d \varphi / d q_{0}$ being computed numerically. Obviously, the results for $f_{\pi}$ are in perfect agreement with the numerical computations.

In order to analyze the influence of the momentum dependence of the vertex function, we display the function $\tilde{f}_{\pi}$, too (thin lines). Since $\tilde{f}_{\pi}$ contains the leading term, Eq. (69), it is not surprising that it is the dominant contribution to $f_{\pi}$. However, for a correct description of the deviations from the weak-coupling limit, $\delta f_{\pi}^{2}$ can be quite important: Whereas for $\Lambda=600 \mathrm{MeV}$, we find that $f_{\pi}$ is well reproduced by $\tilde{f}_{\pi}$, this is not the case for $\Lambda=700 \mathrm{MeV}$. This indicates that the correction due to the contributions arising from the momentum dependence of the vertex function are rather small for $\Lambda=600 \mathrm{MeV}$ and considerably larger for $\Lambda=700 \mathrm{MeV}$.

This result becomes clear if we look at the derivative $d \varphi / d q_{0}$ at $q=0$, which is shown in Fig. 6. For $\Lambda=700 \mathrm{MeV}$ (dashed line) the derivative is nowhere small in the shown region. Therefore, $\delta f_{\pi}^{2}$ can never be neglected, in agreement with our findings in Fig. 5. On the other hand, for $\Lambda=600 \mathrm{MeV}$ (solid line) we find that the derivative is rather small for $\Delta \gtrsim 40 \mathrm{MeV}$, explaining why $\delta f_{\pi}^{2}$ is negligible in this regime. For smaller values of $\Delta$ the correction becomes larger, in agreement with the deviations we found in Fig. 5. In fact, for $\Delta \rightarrow 0$, the derivative even seems to diverge. If it was stronger than $\left(y^{2} \ln y^{2}\right)^{-1}$, the divergence could affect the weakcoupling limit. However, at least numerically, we find that $d \varphi / d q_{0}$ grows much slower than $\left(y^{2} \ln y^{2}\right)^{-1}$, and we 


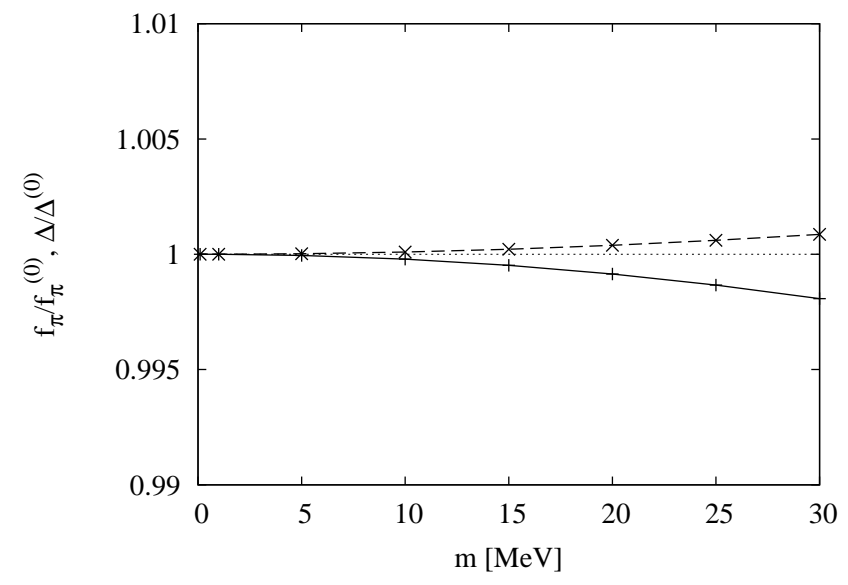

FIG. 7: $f_{\pi}$ (solid line) and $\Delta$ (dashed line) divided by their respective chiral limit values $f_{\pi}^{(0)}$ and $\Delta^{(0)}$ as functions of the common quark mass $m$. The calculations have been performed for $H \Lambda^{2}=1.4$, corresponding to $\Delta^{(0)}=79.1 \mathrm{MeV}$.

therefore conclude that the weak-coupling limit is safe.

\section{B. Equal quark masses}

Next, we study the effect of explicit chiral symmetry breaking through non-vanishing, but equal quark masses $m_{u}=m_{d}=m_{s} \equiv m$. For the cutoff, we choose $\Lambda=$ $600 \mathrm{MeV}$ and keep this value fixed in the remainder of this article.

Before turning to our main focus, i.e., the masses of the Goldstone bosons, we briefly investigate the dependence of the pion decay constant and of the gap parameter $\Delta$ on $m$. The results are displayed in Fig. 7. We find that both quantities depend only very weakly on the quark mass. In the plotted range they vary less than $0.2 \%$. This is much weaker than the $m$ dependence of $f_{\pi}$ in vacuum or of the constituent quark mass in comparable models. In the following discussion we will therefore neglect the distinction between the $m$ dependent gap parameter $\Delta$ and its chiral limit $\Delta^{(0)}$ and often use $\Delta$ in order to characterize the coupling strength.

From Eq. (63), we expect that, to leading order, the masses $m_{M}$ of the Goldstone bosons in the octet depend linearly on $m$,

$$
m_{M}=\sqrt{\frac{8 A}{f_{\pi}^{2}}} m .
$$

This is confirmed by our numerical calculations. In Fig. 8 the values of $m_{M}$ are displayed as functions of the quark mass for three different couplings $H \Lambda^{2}=0.6,1.0$, and 1.4, corresponding to CFL gaps of $12.5 \mathrm{MeV}, 43.2 \mathrm{MeV}$, and $79.1 \mathrm{MeV}$, respectively. As one can see, the results show an almost perfect linear behavior.

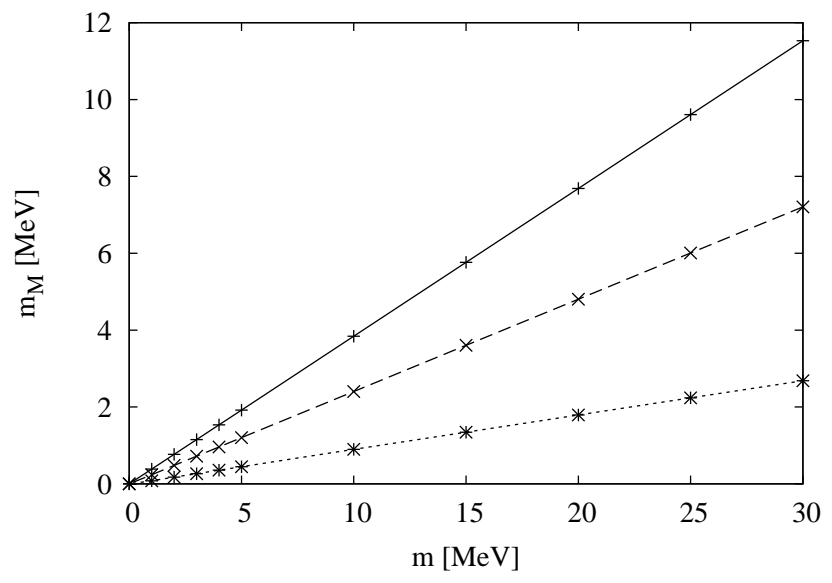

FIG. 8: Masses of the flavored (pseudo-) Goldstone bosons as functions of a common quark mass $m$ for three different couplings. Solid: $H \Lambda^{2}=1.4$ (corresponding to $\Delta=79.1 \mathrm{MeV}$ ), dashed: $H \Lambda^{2}=1.0(\Delta=43.2 \mathrm{MeV})$, dotted: $H \Lambda^{2}=0.6$ $(\Delta=12.5 \mathrm{MeV})$.

The slopes of the straight lines are decreasing with decreasing coupling strength $H$, i.e., with decreasing $\Delta$. This is also expected from the QCD weak-coupling limit, Eq. (67) and Eq. (69) inserted in Eq. (74). However, as discussed in Sect. III B, we expect that the $\Delta$-dependence of the slopes in the NJL model and in weak-coupling QCD is rather different. To study this aspect in detail, we determine the slopes $a$ of the functions $m_{M}(m)=a m$ for different values of $\Delta$ and use our ( $\Delta$ dependent) results for $f_{\pi}$ to obtain $A=\frac{1}{8} a^{2} f_{\pi}^{2}$.

The result, divided by the weak-coupling limit $A_{w c}$ is displayed in Fig. 9 as a function of $\Delta$. The ratios obtained from the fit to the numerically determined meson masses are indicated by the crosses. The solid line corresponds to Eq. (E1), i.e., to the exact analytical solution of Eq. (64), the dashed line indicates the approximate formula Eq. (65). The former is again in perfect agreement with the numerical results.

We see that for small values of $\Delta, A$ is larger than $A_{w c}$. This is due to the logarithmic term discussed in Sec. III B (see Eq. (65) and the subsequent discussion). For large couplings, on the other hand, the NJL-model value of $A$ is considerably smaller than $A_{w c}$, leading to even smaller Goldstone-boson masses than predicted in weak coupling. This has also been found in Ref. [40] within a rather different approach.

\section{Unequal quark masses}

Finally, we study the effect of unequal quark masses. In the upper panel of Fig. 10 the pole positions of the flavored (pseudo-) Goldstone modes are displayed as functions of the strange quark mass, keeping $m_{u}$ and $m_{d}$ fixed. Our numerical results are indicated by the 


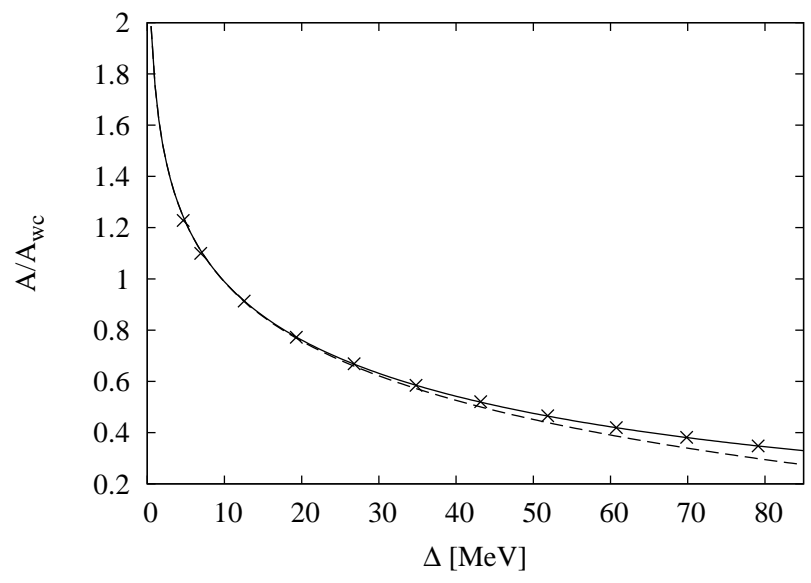

FIG. 9: Ratio of $A$ and $A_{w c}$ as a function of the gap parameter $\Delta$. Points: numerical results; solid line: exact analytical result with $A$ from Eq. (E1); dashed line: approximate result with $A$ from Eq. (65).

points. For practical reasons, we have chosen a relatively strong diquark coupling $H \Lambda^{2}=1.4$ (corresponding to $\Delta=79.1 \mathrm{MeV}$ ) and a relatively large value of $30 \mathrm{MeV}$ for $m_{u}$ and $m_{d}$, in order to have not too small meson masses. As one can see, the poles which are degenerate for equal masses split into three branches, corresponding to different strangeness, i.e., pions $(S=0)$, kaons $(S=+1)$, and antikaons $(S=-1)$. On the other hand, since we have chosen $m_{u}=m_{d}$, the different isospin states of these modes, i.e., $\pi^{-}$and $\pi^{+}, K^{+}$and $K^{0}$, and $K^{-}$and $\bar{K}^{0}$ remain degenerate.

Our results can be analyzed in terms of the parameterization given in Eq. (39). Each mode $T^{(i)}$ has two poles, which for $\vec{q}=0$ are located at

$$
q_{0}= \pm m_{i}-\mu_{i} \equiv \omega_{i}^{ \pm} .
$$

We can thus extract the meson masses and chemical potentials as

$$
m_{i}=\frac{1}{2}\left(\omega_{i}^{+}-\omega_{i}^{-}\right), \quad \mu_{i}=-\frac{1}{2}\left(\omega_{i}^{+}+\omega_{i}^{-}\right) .
$$

The resulting functions are displayed in the two lower panels of Fig. 10. The masses are moderately rising with $m_{s}$ and exhibit an "inverse ordering" $\left(m_{K}<m_{\pi}\right)$, as predicted first in Ref. [23]. Also note that kaons and antikaons have the same masses. For the meson chemical potentials, on the other hand, we find $\mu_{K}=-\mu_{\bar{K}}$, as it should be, and $\mu_{\pi}=0$.

These results can be compared with those derived in Refs. [23] and [26, 27] in an effective field theory (EFT) approach. For $m_{u}=m_{d} \equiv m_{q}$, they read

$$
m_{\pi}=\sqrt{\frac{8 A}{f_{\pi}^{2}} m_{s} m_{q}}, \quad m_{K}=m_{\bar{K}}=\sqrt{\frac{4 A}{f_{\pi}^{2}} m_{q}\left(m_{q}+m_{s}\right)},
$$
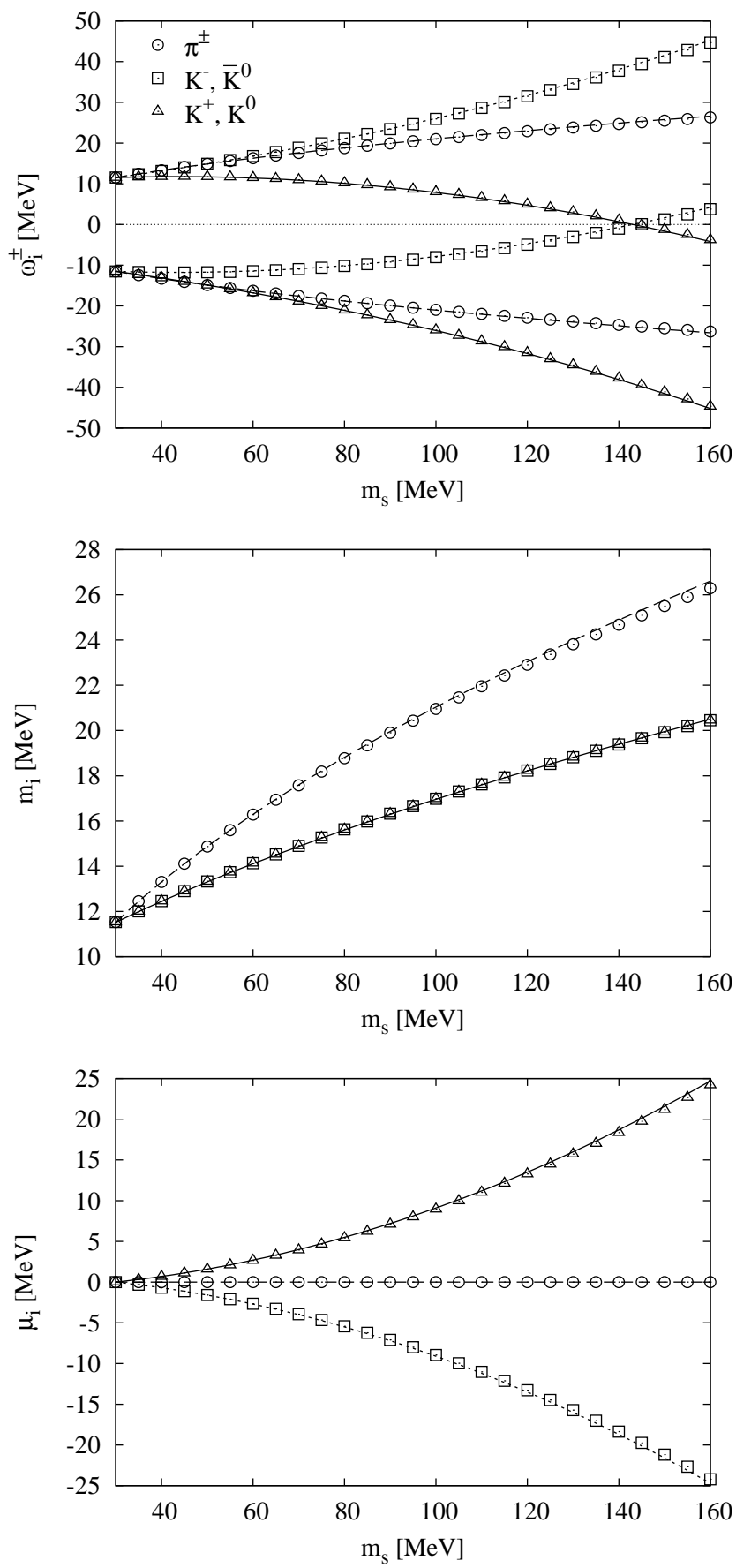

FIG. 10: Properties of the flavored Goldstone modes as functions of the strange quark mass for $m_{u}=m_{d}=30 \mathrm{MeV}$ and $H \Lambda^{2}=1.4(\Delta=79.1 \mathrm{MeV}):$ pole positions of the T-matrix at $\vec{q}=0$ (upper panel), meson masses (center), and meson chemical potentials (lower panel). The various points indicate the numerical results: $\pi^{ \pm}$(circles), $K^{+}$and $K^{0}$ (triangles), and $K^{-}$and $\bar{K}^{0}$ (squares). The lines correspond to the predictions from Eqs. (75), (77), and (78). 
and

$$
\mu_{\pi^{ \pm}}=0, \quad \mu_{K^{ \pm}}=\mu_{K^{0}, \bar{K}^{0}}= \pm \frac{m_{s}^{2}-m_{q}^{2}}{2 \mu} .
$$

For $A$ and $f_{\pi}$ we insert the NJL-model values obtained in Sects. IV A and IV B in the limit of vanishing quark masses. The resulting functions are indicated by the lines in Fig. 10. Obviously, they agree almost perfectly with the numerical calculations. Note, however, that the agreement would not be good if we had used the weak-coupling results for $A$ and $f_{\pi}$.

When $\mu_{i}$ reaches the value of $m_{i}$,

$$
\mu_{i}\left(m_{q}, m_{s}^{c r i t}\right)=m_{i}\left(m_{q}, m_{s}^{c r i t}\right),
$$

$\omega_{i}^{+}$vanishes and meson condensation sets in. For the chosen parameters, this occurs in the kaon branch $\left(K^{+}\right.$and $K^{0}$ ) at a critical strange quark mass $m_{s}^{\text {crit }} \approx 145 \mathrm{MeV}$. For higher strange quark masses the CFL phase is no longer the correct ground state and the shown results have no physical meaning.

Inserting the EFT expressions Eqs. (77) and (78) into Eq. (79) one finds that the critical strange quark mass for kaon condensation is approximately given by [26]

$$
m_{s}^{c r i t} \approx\left(\frac{16 \mu^{2} A}{f_{\pi}^{2}}\right)^{1 / 3} m_{q}^{1 / 3}
$$

which becomes exact in the limit $m_{q} \rightarrow 0$. For $m_{q}=0$ this implies that kaon condensation is favored for arbitrarily small strange quark masses. One might expect that this is also the case in our model, after we found good agreement with the EFT predictions in Fig. 10.

On the other hand, this would contradict an earlier NJL-model study [40], where a nonzero critical strange quark mass was found, even for $m_{q}=0$. This was concluded, without explicit construction of the Goldstone bosons, by studying the stability of the CFL ground state against partially rotating the scalar diquark condensates into pseudoscalar ones. Thus, with our present approach, we can check this result from a different perspective. Since in Ref. [40] indications were found that the deviations from the EFT predictions are due to terms of higher order in the interaction, we perform our analysis using a rather strong diquark coupling, $H \Lambda^{2}=1.7$, corresponding to $\Delta=107.9 \mathrm{MeV}$.

In Fig. 11, the critical strange quark mass for kaon condensation is displayed as a function of the third root of $m_{q}$. The NJL-model results are indicated by the crosses. We also show the solution of Eq. (79) for the EFT masses and chemical potentials Eqs. (77) and (78) (solid line) and the approximate solution Eq. (80) (dashed).

We find that the NJL points are always above the EFT predictions (solid line). However, while the deviations are small for $m_{q} \gtrsim 1 \mathrm{MeV}$, they become essential for smaller values of $m_{q}$. In particular, we confirm that $m_{s}^{\text {crit }}$ does not vanish at $m_{q}=0$ but goes to a finite value, which is about $21 \mathrm{MeV}$ in our example.

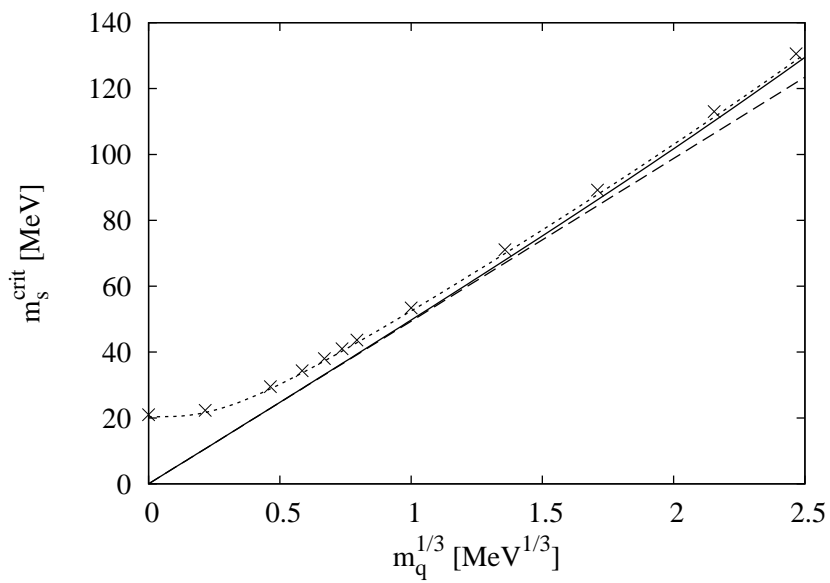

FIG. 11: Critical strange quark mass $m_{s}^{\text {crit }}$ for kaon condensation as a function of $m_{q}^{1 / 3}$, where $m_{q} \equiv m_{u}=m_{d}$ : NJLmodel results (crosses), EFT predictions using Eqs. (77) and (78) (solid line), and approximate solution Eq. (80). The dotted line is based on Eq. (81) with $a_{s}=0.0203$. The NJLmodel calculations have been performed with $H \Lambda^{2}=1.7$, corresponding to $\Delta=107.9 \mathrm{MeV}$.

To understand this behavior, we analyze the poles of the T-matrix in the kaon channel for $m_{q}=0$ as functions of $m_{s}$. In Fig. 12 the NJL-model results (crosses) are compared with the EFT predictions (solid lines). As before, the pole positions (upper panel) can be interpreted in terms of kaon masses (center) and chemical potentials (lower panel). It turns out that the latter are in fair agreement with Eq. (78). On the other hand, while Eq. (77) predicts the kaon masses to be zero for $m_{q}=0$, we find that $m_{K}$ is in general non-zero and rises linearly with $m_{s}$. As a consequence, the pole position $\omega_{K}^{+}$is not degenerate with $\omega_{K}^{-}$(cf. Eq. (75)) and first rises with $m_{s}$. Hence, kaon condensation does not occur at arbitrarily small values of $m_{s}$ but only for $m_{s} \gtrsim 21 \mathrm{MeV}$, as already seen in Fig. 11.

It should be noted, however, that, although the linear rise of $m_{K}$ with $m_{s}$ is qualitatively different from Eq. (77), the slope is very small on a quantitative scale. In our example, a linear fit $m_{K}=a_{s} m_{s}$ yields $a_{s}=0.0203$, which is an order of magnitude smaller than the slope in Eq. (77), $\sqrt{4 A / f_{\pi}^{2}}=0.347$. Moreover, by varying the coupling strength, we found numerically that $a_{s}$ depends quadratically on $\Delta$. This is consistent with our expectation that the effect corresponds to a higher-order correction in the interaction and only becomes visible when the leading order, Eq. (77), is artificially suppressed by choosing very small values of $m_{q}$. In fact, for any realistic value of $m_{u}$ and $m_{d}$ the correction is quite irrelevant.

Also note that our results are somewhat complementary to Ref. [52], where corrections to the effective kaon chemical potential have been discussed. However, it was found there that these corrections are of the order of $\left(m_{s}^{2} / 2 \mu \Delta\right)^{2}$, which is completely negligible in all of our 

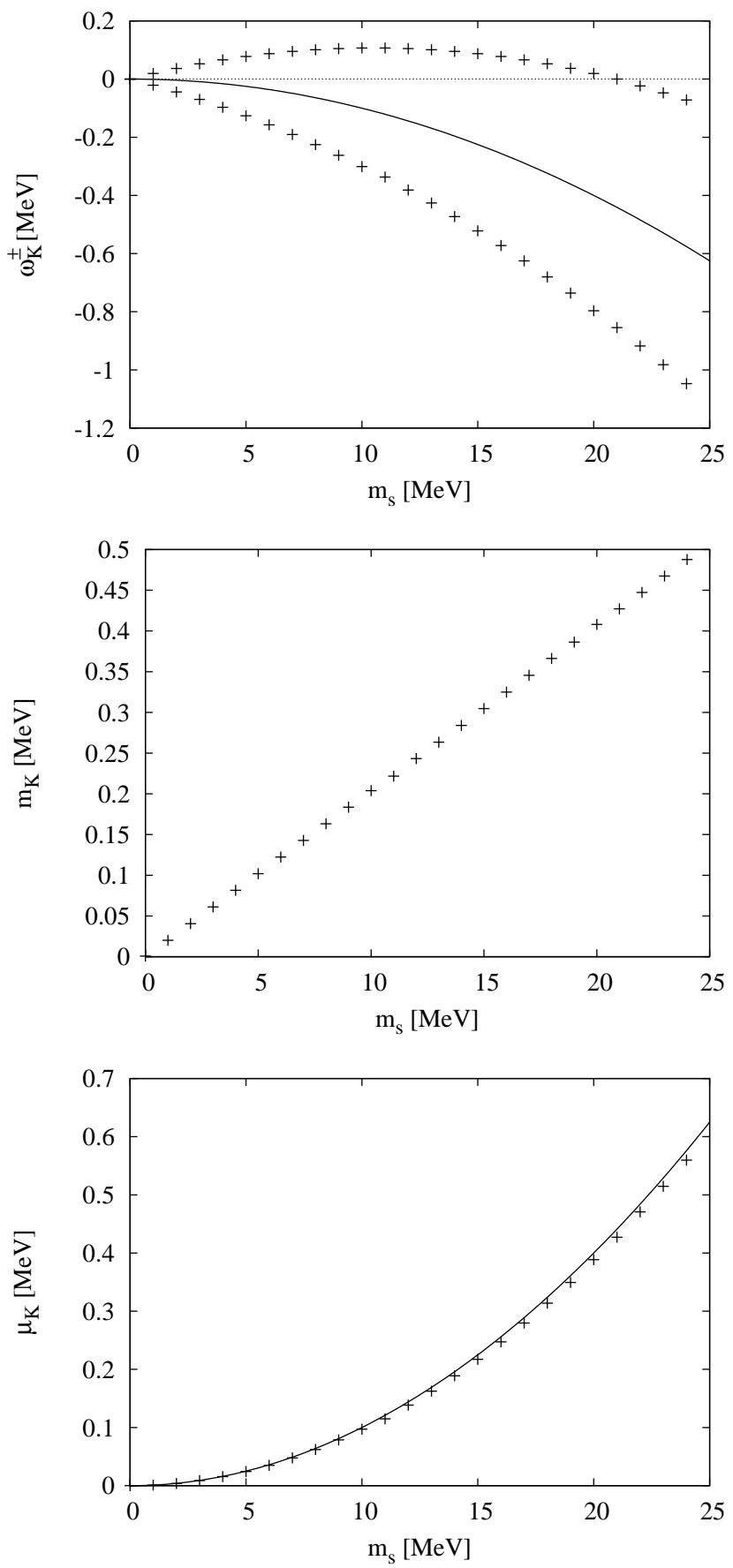

FIG. 12: Kaon $\left(K^{+}\right.$and $\left.K^{0}\right)$ properties as functions of the strange quark mass for $m_{u}=m_{d}=0$ and $H \Lambda^{2}=1.7(\Delta=$ 107.9 MeV): pole positions of the T-matrix at $\vec{q}=0$ (upper panel), kaon masses (center), and kaon chemical potentials (lower panel). The crosses indicate the numerical results, the lines correspond to the predictions from Eqs. (75), (77), and (78). Note that from Eq. (77) $m_{K}$ is expected to vanish. examples.

We may finally combine Eq. (77) with our numerical findings for $m_{q}=0$ by parameterizing

$$
m_{K}=\sqrt{\frac{4 A}{f_{\pi}^{2}} m_{q}\left(m_{q}+m_{s}\right)+a_{s}^{2} m_{s}^{2}} .
$$

Equating this with the kaon chemical potential, Eq. (78), we obtain the dotted line in Fig. 11 for the critical strange quark mass. It is obviously in good agreement with our numerical results. The tiny deviations for small values of $m_{q}$ can be explained by the fact that the kaon chemical potential in Fig. 12 is slightly overestimated by Eq. (78). A similar effect could also play a role at larger $m_{q}$, but there might be other terms as well.

\section{SUMMARY AND CONCLUSIONS}

We have studied the properties of pseudoscalar Goldstone boson excitations in the color-flavor-locked phase within an NJL-type model. To that end we solved the Bethe-Salpeter equation in RPA. So far we have only included quark-quark interactions such that our Goldstone boson states are in fact superpositions of diquark and di-hole states.

Our results are consistent with the model independent predictions of the low-energy effective theory, i.e., with those predictions which only depend on the symmetry breaking pattern. We found, however, deviations from the values for the constants appearing in the LEET, as for instance the pion decay constant $f_{\pi}$, obtained in the weak-coupling limit. In fact, it was the main motivation of this paper to locate such deviations and to understand their origins. In several cases this could even be done analytically. Although these model results are in general not universal, they may give important hints about to what extent the weak-coupling results can be trusted in the intermediate-density regime and where to expect major deviations.

The weak-coupling limit for $f_{\pi}$ [23] is correctly reproduced in the chiral limit in zeroth order in the gap parameter $\Delta$. This must be the case since this result is universal, i.e., independent of the specific choice of the interaction. For $\Delta$ not being small we found deviations, typically of the order of a few percent. We have shown that this is an effect of higher order in the gap parameter. In this context, the momentum dependence of the dressed vertex function plays an interesting role.

Next, we discussed the masses of the Goldstone bosons in the limit of equal quark masses. In agreement with the LEET prediction, we found that the meson masses behave linearly in the quark masses. However, the corresponding coefficient $A$ does not agree with the weakcoupling result obtained in HDET [23]. In the limit $\Delta \rightarrow 0$, this should be viewed as an artifact of the NJL model. Probably more relevant are the deviations at large 
values of $\Delta$ where we found the meson masses to be considerably smaller than predicted by the weak-coupling formula.

Finally, we have studied the case of unequal quark masses. In general, we found a very good agreement with the LEET prediction for the meson masses and effective chemical potentials, Eqs. (77) and (78). In particular, we found $m_{K}<m_{\pi}$, i.e., an inverse meson mass ordering as predicted in Ref. [23]. We also confirmed that the strange quark mass acts as an effective strangeness chemical potential, eventually leading to kaon condensation at sufficiently large values of $m_{s}$ Refs. [26, 27]. However, even quantitatively, our model results are in an almost perfect agreement with Eqs. (77) and (78) if the constants $A$ and $f_{\pi}$ entering Eq. (77) are taken from our preceding NJL-model studies in the limit of vanishing quark masses. Since these values do not agree with the weak-coupling limit, as discussed above, our meson masses are in general smaller than those obtained with the weak-coupling coefficients.

In the limit of vanishing light quark masses, we found a qualitative difference. The critical strange quark mass for the onset of kaon condensation does not vanish with the cubic root of light quark masses but attains a nonzero value. We identified this numerically as a higher order effect on the kaon mass, adding a $\Delta^{2} m_{s}$ dependence at low light quark masses.

This paper should be seen as the basis for further studies of the Goldstone boson dynamics in cold dense quark matter at non-asymptotic densities where deviations from the weak-coupling limit become visible. As already mentioned, our simple Lagrangian does not include quark-antiquark interactions, which, although subdominant, can give important corrections to the pseudoscalar meson masses. Moreover, we miss instanton effects, which have been shown to be important $[28,34]$. The ultimate goal is to include the back reaction of the Goldstone bosons on the phase structure of color superconducting quark matter [54]. In the intermediate density regime, the ratio of the gap parameter and the Fermi energy is of the order of 0.25 , such that the Goldstone boson excitations can have a significant effect on the ground state properties.

\section{Acknowledgments}

We thank M. Ruggieri for useful comments. This work has been supported in part by the BMBF under contract 06DA123 and by the Helmholtz-University Young Investigator Grant VH-NG-332.

\section{APPENDIX A: GAP EQUATIONS}

The Dyson equation shown in Fig. 1 reads

$$
S(p)=S_{0}(p)+S_{0}(p) \hat{\Sigma} S(p) .
$$

Solving for the self-energy, one obtains

$$
\hat{\Sigma}=S_{0}^{-1}(p)-S^{-1}(p),
$$

where

$$
S_{0}^{-1}(p)=\left(\begin{array}{cc}
\not p+\hat{\mu} \gamma^{0}-\hat{m} & 0 \\
0 & \not p-\hat{\mu} \gamma^{0}-\hat{m}
\end{array}\right)
$$

is the inverse bare quark propagator, while $S^{-1}$ is the inverse dressed propagator defined in Eq. (16). Thus,

$$
\hat{\Sigma}=\left(\begin{array}{cc}
0 & -\sum_{A=2,5,7} \Delta_{A} \gamma_{5} \tau_{A} \lambda_{A} \\
\sum_{A=2,5,7} \Delta_{A}^{*} \gamma_{5} \tau_{A} \lambda_{A} & 0
\end{array}\right) .
$$

On the other hand, $\hat{\Sigma}$ can be evaluated diagrammatically. In Hartree approximation, it corresponds to the quark loop in Fig. 1 and is given by

$$
\hat{\Sigma}=4 i H \Gamma_{i} \int \frac{d^{4} k}{(2 \pi)^{4}} \frac{1}{2} \operatorname{Tr}\left[\bar{\Gamma}_{i} S(k)\right] .
$$

Comparing the two expressions for $\hat{\Sigma}$, we can read off the following gap equations:

$$
\Delta_{A}=4 H \int \frac{d^{4} k}{(2 \pi)^{4}} \frac{1}{2} \operatorname{Tr}\left[\Gamma_{A A}^{s \downarrow} S(k)\right] .
$$

We also see that the contributions of scalar vertices with $A \neq A^{\prime}$ and of pseudoscalar vertices should vanish to be consistent. Using the explicit expression for the dressed propagator (see Appendix C), it can be shown that this is indeed the case.

\section{APPENDIX B: CHIRAL WARD-TAKAHASHI IDENTITY}

In this appendix, we demonstrate that the dressed vertex functions and the dressed quark propagator are consistent with the chiral Ward-Takahashi identity $(\chi \mathrm{WTI})$ in the sense that, if the $\chi$ WTI holds, we recover the gap equation. As in Sect. III, we restrict ourselves to the case of equal quark masses.

From Eqs. (44) and (47) we obtain

$$
\begin{aligned}
q_{\mu} \Gamma_{5 j}^{\mu}(p ; q) & +2 m i \Gamma_{5, j}(p ; q) \\
=\left((q-2 m) \gamma_{5} t_{j}\right)_{N G} & \\
\quad+4 i H & \Gamma_{i} \int \frac{d^{4} k}{(2 \pi)^{4}} \frac{1}{2} \operatorname{Tr}\left[\bar{\Gamma}_{i} S(k+q)\right. \\
& \left.\times\left(q_{\mu} \Gamma_{5 j}^{\mu}(k ; q)+2 m i \Gamma_{5, j}(k ; q)\right) S(k)\right] .
\end{aligned}
$$


Hence, imposing the $\chi$ WTI, Eq. (52), one gets

$$
\begin{aligned}
& S^{-1}(p+q)\left(\gamma_{5} t_{j}\right)_{N G}+\left(\gamma_{5} t_{j}\right)_{N G} S^{-1}(p) \\
& =\left((q-2 m) \gamma_{5} t_{j}\right)_{N G} \\
& +4 i H \Gamma_{i} \int \frac{d^{4} k}{(2 \pi)^{4}} \frac{1}{2} \operatorname{Tr}\left[\left(\bar{\Gamma}_{i}\left(\gamma_{5} t_{j}\right)_{N G}\right.\right. \\
& \left.\left.+\left(\gamma_{5} t_{j}\right)_{N G} \bar{\Gamma}_{i}\right) S(k)\right]
\end{aligned}
$$

Using Eq. (16) with equal masses and gap parameters, one finds that the diagonal Nambu-Gorkov components are equal to the first term on the r.h.s., and one is left with

$$
\begin{gathered}
\left(\begin{array}{cc}
0 & \Delta \sum_{A=2,5,7}\left(\tau_{A} t_{j}^{T}+t_{j} \tau_{A}\right) \lambda_{A} \\
-\Delta^{*} \sum_{A=2,5,7}\left(\tau_{A} t_{j}+t_{j}^{T} \tau_{A}\right) \lambda_{A} & 0
\end{array}\right) \\
=4 i H \Gamma_{i} \int \frac{d^{4} k}{(2 \pi)^{4}} \frac{1}{2} \operatorname{Tr}\left[\left(\bar{\Gamma}_{i}\left(\gamma_{5} t_{j}\right)_{N G}\right.\right. \\
\left.\left.+\left(\gamma_{5} t_{j}\right)_{N G} \bar{\Gamma}_{i}\right) S(k)\right] .
\end{gathered}
$$

Next we compute the sums over $A$ on the l.h.s. for any of the flavor operators $t_{j}$. For instance, for $t_{j}=t_{\pi^{+}}=$ $\frac{\tau_{1}+i \tau_{2}}{2 \sqrt{2}}$ we find that the l.h.s. is equal to $\frac{1}{\sqrt{2}}\left(\Delta \Gamma_{57}^{p \uparrow}+\right.$ $\left.\Delta^{*} \Gamma_{75}^{p \downarrow}\right)$. We conclude that the r.h.s. must vanish for all $\Gamma_{i}$, except for $\Gamma_{i}=\Gamma_{57}^{p \uparrow}$ and $\Gamma_{i}=\Gamma_{75}^{p \downarrow}$. In the first case, we have $\bar{\Gamma}_{i}\left(\gamma_{5} t_{\pi^{+}}\right)_{N G}+\left(\gamma_{5} t_{\pi^{+}}\right)_{N G} \bar{\Gamma}_{i}=-\frac{i}{\sqrt{2}} \Gamma_{77}^{s \downarrow}$. Thus by comparison with the l.h.s. we obtain

$$
\Delta=4 H \int \frac{d^{4} k}{(2 \pi)^{4}} \frac{1}{2} \operatorname{Tr}\left[\Gamma_{77}^{s \downarrow} S(k)\right],
$$

in agreement with one of the gap equations (A6) for the case of equal masses. The two other equations can be derived analogously, if we evaluate Eq. (B3) for $t_{j}=t_{K^{+}}$ or $t_{j}=t_{K^{0}}$. Moreover, the fact that most $\Gamma_{i}$ must not contribute to the r.h.s. for a given $t_{j}$ can be used to show that scalar operators with $A \neq A^{\prime}$ and pseudoscalar operators do not contribute to the gap equation.

\section{APPENDIX C: DRESSED QUARK PROPAGATOR}

\section{General case}

The dressed quark propagator $S(p)$ is the inverse of the inverse quark propagator, defined in Eq. (16). Following standard methods (see, e.g., Refs. [47-50]), we write

$$
S^{-1}(p) \equiv S^{-1}\left(p^{0}, \vec{p}\right)=\gamma^{0}\left(p^{0}-A(\vec{p})\right),
$$

where $A(\vec{p})$ is a hermitian $72 \times 72$ matrix, which does not depend on $p^{0}$. Thus $A$ can always be diagonalized, i.e., we can find a unitary matrix $U(\vec{p})$, so that

$$
A(\vec{p})=U^{\dagger}(\vec{p}) D(\vec{p}) U(\vec{p}),
$$

with

$$
D(\vec{p})=\left(\begin{array}{ccc}
\varepsilon_{1}(\vec{p}) & & 0 \\
& \ddots & \\
0 & & \varepsilon_{72}(\vec{p})
\end{array}\right)
$$

being a diagonal matrix with eigenvalues $\varepsilon_{1}, \ldots \varepsilon_{72}$. It can be shown that all eigenvalues are two-fold degenerate, and for each eigenvalue $\varepsilon_{i}$, there is a counterpart $-\varepsilon_{i}$ in the spectrum. This means, there are basically 18 independent eigenvalues. Moreover, part of the diagonalization is trivial because the matrix $A$ can be brought into block diagonal form by reordering of lines and columns. The remaining blocks are in general diagonalized numerically.

Combining Eqs. (C1) and (C3), the propagator is finally given by

$$
S(p)=U^{\dagger}(\vec{p})\left(\begin{array}{ccc}
\frac{1}{p^{0}-\varepsilon_{1}(\vec{p})} & & 0 \\
& \ddots & \\
0 & & \frac{1}{p^{0}-\varepsilon_{72}(\vec{p})}
\end{array}\right) U(\vec{p}) \gamma^{0} .
$$

\section{Equal quark masses}

In the limit of an exact $S U(3)$ symmetry, we can give a closed expression for the quark propagator. Straight forward inversion of Eq. (16) for $m_{u}=m_{d}=m_{s}=m$ and $\Delta_{22}=\Delta_{55}=\Delta_{77}=\Delta$ yields

$$
S=\left(\begin{array}{ll}
S_{11} & S_{12} \\
S_{21} & S_{22}
\end{array}\right)
$$

with

$$
S_{21}=\Delta^{*} \frac{\not p_{-}+m}{x_{-}} \gamma_{5} \sum_{A=2,5,7} \tau_{A} \lambda_{A} S_{11}
$$

and

$$
S_{11}=\left[\not p_{+}-m-\frac{|\Delta|^{2}}{x_{-}}\left(\not p_{-}-m\right) \sum_{A, A^{\prime}=2,5,7} \tau_{A} \tau_{A^{\prime}} \lambda_{A} \lambda_{A^{\prime}}\right]^{-1}
$$

where we have introduced the notations

$$
p_{ \pm}=p \pm \mu \gamma^{0}, \quad x_{ \pm}=p_{ \pm}^{2}-m^{2} .
$$

$S_{22}$ and $S_{12}$ are obtained from $S_{11}$ and $S_{21}$, respectively, under the exchange $\mu \leftrightarrow-\mu$ and $\Delta \leftrightarrow-\Delta^{*}$.

The matrices $S_{i j}$ are $36 \times 36$ matrices representing the normal $(i=j)$ and anomalous $(i \neq j)$ Nambu-Gorkov components of $S$. $S_{11}$ can explicitly be written as

$$
S_{11}=S_{-}+\frac{1}{6}\left(T_{-}-S_{-}\right) \sum_{a=0}^{8} \tau_{a} \lambda_{a},
$$


with

$$
S_{ \pm}=\frac{x_{ \pm}\left(\not p_{\mp}+m\right)-|\Delta|^{2}\left(\not p_{ \pm}+m\right)}{\left(p_{0}^{2}-E_{8}^{-2}\right)\left(p_{0}^{2}-E_{8}^{+2}\right)}
$$

corresponding to the eigenvalue $\Delta$ of the gap matrix, and

$$
T_{ \pm}=\frac{x_{ \pm}\left(\not p_{\mp}+m\right)-4|\Delta|^{2}\left(\not p_{ \pm}+m\right)}{\left(p_{0}^{2}-E_{1}^{-2}\right)\left(p_{0}^{2}-E_{1}^{+2}\right)}
$$

corresponding to the eigenvalue $2 \Delta$ of the gap matrix. The octet and singlet dispersion relations for particles $(-)$ and antiparticles $(+)$ are given by

$$
E_{8}^{\mp}=\sqrt{\left(\sqrt{\vec{p}^{2}+m^{2}} \mp \mu\right)^{2}+|\Delta|^{2}}
$$

and

$$
E_{1}^{\mp}=\sqrt{\left(\sqrt{\vec{p}^{2}+m^{2}} \mp \mu\right)^{2}+4|\Delta|^{2}},
$$

respectively.

\section{APPENDIX D: PION DECAY CONSTANT IN THE CHIRAL LIMIT}

In this appendix we derive a semi-analytical expression for the pion decay constant in the chiral limit, which is used in Sect. IV A to discuss the deviations from the weak coupling limit. The $\eta^{\prime}$ decay constant can be obtained in a similar way but we do not discuss this here.

Starting point is Eq. (43) for the (time-like) decay constant in the case of equal quark masses. In the chiral limit, we have to evaluate this formula at $m_{i}=0$, Since both sides of this equation vanish in this limit, this means that the decay constant is given by the derivative of the r.h.s. with respect to $q_{0}$. Moreover, we can employ the "Goldberger-Treiman relation", Eq. (55), to eliminate the coupling constant $g_{i}$. We then obtain

$$
\begin{aligned}
f_{i}^{2}=-\Delta\left(\frac{d}{d q_{0}} \int \frac{d^{4} k}{(2 \pi)^{4}}\right. & \\
& \left.\frac{1}{2} \operatorname{Tr}\left[\bar{\Gamma}_{i}^{\prime}(q) S(k+q)\left(\gamma^{0} \gamma_{5} t_{i}\right)_{N G} S(k)\right]\right)\left.\right|_{q=0} .
\end{aligned}
$$

Here we have indicated explicitly that the vertex function $\Gamma_{i}^{\prime}$ depends on the momentum (see Eq. (60)) and is therefore subject to the derivative. We may thus write

$$
f_{i}^{2}=\tilde{f}_{i}^{2}+\delta f_{i}^{2}
$$

where

$$
\begin{aligned}
& \tilde{f}_{i}^{2}= \\
& -\Delta \int \frac{d^{4} k}{(2 \pi)^{4}} \frac{1}{2} \operatorname{Tr}\left[\left.\bar{\Gamma}_{i}^{\prime}(0) \frac{d S(k+q)}{d q_{0}}\right|_{q=0}\left(\gamma^{0} \gamma_{5} t_{i}\right)_{N G} S(k)\right]
\end{aligned}
$$

corresponds to the contribution where the derivative acts on the propagator, while

$$
\begin{aligned}
& \delta f_{i}^{2}= \\
& -\Delta \int \frac{d^{4} k}{(2 \pi)^{4}} \frac{1}{2} \operatorname{Tr}\left[\left.\frac{d \bar{\Gamma}_{i}^{\prime}(q)}{d q_{0}}\right|_{q=0} S(k)\left(\gamma^{0} \gamma_{5} t_{i}\right)_{N G} S(k)\right]
\end{aligned}
$$

corresponds to the contribution where the derivative acts on the vertex function.

The evaluation of $\tilde{f}_{i}^{2}$ is tedious, but straight forward. Inserting $\Gamma_{i}^{\prime}(0)=\Gamma_{i}^{\prime(0)}$ from Eqs. (57) or (58), respectively, $t_{i}$ from Table I as well as the expressions for the quark propagator given in appendix C 2 in the chiral limit, the result for the octet mesons ("pions") reads

$$
\begin{aligned}
& \tilde{f}_{\pi}^{2}= \frac{\mu^{2}}{216 \pi^{2}} \\
&\left\{\frac { 2 } { y ^ { 2 } } \left[\left(\alpha_{1}^{+}-\alpha_{8}^{+}\right)(x+1)\left(3 x^{2}-2 x+1\right)\right.\right. \\
&\left.+\left(\alpha_{1}^{-}-\alpha_{8}^{-}\right)(x-1)\left(3 x^{2}+2 x+1\right)\right] \\
&-\alpha_{8}^{+}(9 x-31)-\alpha_{8}^{-}(9 x+31) \\
&-16 \alpha_{1}^{+}+16 \alpha_{1}^{-}-45 \beta \\
&+24\left[\ln \left(\alpha_{1}^{+}+x+1\right)+\ln \left(\alpha_{1}^{-}+x-1\right)\right. \\
&\left.\quad-\ln \left(\alpha_{8}^{+}+x+1\right)-\ln \left(\alpha_{8}^{-}+x-1\right)\right] \\
&+45\left[\frac{x+1}{\alpha_{8}^{+}}+\frac{x-1}{\alpha_{8}^{-}}+\frac{1}{\beta}\right] \\
&-48 \ln 2-54 y^{2} \ln y^{2} \\
&+54 y^{2}\left[\ln \left(\alpha_{8}^{+}+x+1\right)+\ln \left(\alpha_{8}^{-}+x-1\right)\right] \\
&\left.-45 y^{2}\left[\frac{x-1}{\alpha_{8}^{+}}+\frac{x+1}{\alpha_{8}^{-}}-\frac{1}{\beta}\right]\right\},
\end{aligned}
$$

where the 3-momentum integral has been regularized by a cutoff $\Lambda$ (as in the numerical calculations) and we have introduced the abbreviations

$$
\alpha_{1}^{ \pm}=\sqrt{(x \pm 1)^{2}+4 y^{2}}, \quad \alpha_{8}^{ \pm}=\sqrt{(x \pm 1)^{2}+y^{2}},
$$

and

$$
\beta=\sqrt{1+y^{2}} .
$$

Moreover, $x=\frac{\Lambda}{\mu}$ and $y=\frac{\Delta}{\mu}$, as defined in Eq. (66).

The evaluation of $\delta f_{i}^{2}$ is more difficult because we need to know the derivative of the vertex function. For the flavored mesons this is encoded in the mixing angle $\varphi(q)$, cf. Eq. (60). We can therefore write

$$
\left.\frac{d \bar{\Gamma}_{i}^{\prime}(q)}{d q_{0}}\right|_{q=0}=\left.\frac{d \varphi(q)}{d q_{0}}\right|_{q=0} \bar{\Gamma}_{i}^{\prime \perp}
$$

where

$$
\Gamma_{i}^{\prime \perp}=\left.\frac{\partial \Gamma_{i}^{\prime}}{\partial \varphi}\right|_{\varphi=\frac{\pi}{4}}
$$


is the vertex of the orthogonal state with the same quantum numbers as the meson $i$ (e.g., $\Gamma_{\pi^{+}}^{\perp \perp}=-\frac{i}{\sqrt{2}}\left(\Gamma_{57}^{p \uparrow}+\right.$ $\left.\left.\Gamma_{75}^{p \downarrow}\right)\right)$. Hence

$$
\begin{aligned}
& \delta f_{i}^{2}= \\
& -\left.\Delta \int \frac{d^{4} k}{(2 \pi)^{4}} \frac{1}{2} \operatorname{Tr}\left[\bar{\Gamma}_{i}^{\prime \perp} S(k)\left(\gamma^{0} \gamma_{5} t_{i}\right)_{N G} S(k)\right] \frac{d \varphi(q)}{d q_{0}}\right|_{q=0} .
\end{aligned}
$$

Evaluating this part for the octet mesons, one finally obtains

$$
\begin{aligned}
\delta f_{\pi}^{2}= & \frac{\mu^{2}}{108 \pi^{2}} \\
\{ & -108 y^{2} \ln y^{2}-48 y^{2} \ln 2 \\
& +2\left(\alpha_{1}^{+}-\alpha_{8}^{+}\right)\left(x^{2}-x+1\right) \\
& -2\left(\alpha_{1}^{-}-\alpha_{8}^{-}\right)\left(x^{2}+x+1\right) \\
& +4 y^{2}\left(\alpha_{8}^{+}-\alpha_{8}^{-}\right)-16 y^{2}\left(\alpha_{1}^{+}-\alpha_{1}^{-}\right) \\
& +12 y^{2}\left[2 \ln \left(\alpha_{1}^{-}+x-1\right)+2 \ln \left(\alpha_{1}^{+}+x+1\right)\right. \\
& \left.\quad+7 \ln \left(\alpha_{8}^{-}+x-1\right)+7 \ln \left(\alpha_{8}^{+}+x+1\right)\right] \\
& -45 y^{2}\left[\frac{x^{2}+4 x+2}{\alpha_{8}^{+}}-\frac{x^{2}-4 x+2}{\alpha_{8}^{-}}\right] \\
& \left.-90 y^{4}\left(\frac{1}{\alpha_{8}^{+}}-\frac{1}{\alpha_{8}^{-}}\right)\right\}\left.\mu \frac{d \varphi(q)}{d q_{0}}\right|_{q=0} .
\end{aligned}
$$

One could also try to derive an analytical expression for the derivative $\left.\frac{d \varphi(q)}{d q_{0}}\right|_{q=0}$ from the polarization-loop matrix Eq. (28), but this is beyond the scope of this paper.

\section{APPENDIX E: EXACT FORMULA FOR $A$}

The exact result of Eq. (64) is given by

$$
\begin{aligned}
& A=\frac{\Delta^{2}}{384 \pi^{2}} \\
& \left\{-48 \ln y^{2}-32 \ln 2+4\left(\alpha_{1}^{-}-\alpha_{1}^{+}\right)+44\left(\alpha_{8}^{-}-\alpha_{8}^{+}\right)\right. \\
& +\left(16+3 y^{2}\right)\left[\ln \left(\alpha_{1}^{-}+x-1\right)+\ln \left(\alpha_{1}^{+}+x+1\right)\right] \\
& +\left(32-3 y^{2}\right)\left[\ln \left(\alpha_{8}^{-}+x-1\right)+\ln \left(\alpha_{8}^{+}+x+1\right)\right] \\
& -6 y^{2} \ln 2 \\
& +9 y^{2} \frac{\beta_{2}}{\beta_{3}}\left[\ln \left[4(x+1)+\alpha_{1}^{+} \beta_{2} \beta_{3}-(3 x-13) y^{2}\right]\right. \\
& -\ln \left[4(x-1)-\alpha_{1}^{-} \beta_{2} \beta_{3}-(3 x+13) y^{2}\right] \\
& +\ln \left[4(x+1)+\alpha_{8}^{+} \beta_{2} \beta_{3}+(3 x+7) y^{2}\right] \\
& \left.\left.-\ln \left[4(x-1)-\alpha_{8}^{-} \beta_{2} \beta_{3}+(3 x-7) y^{2}\right]\right]\right\} \text {, }
\end{aligned}
$$

with $\alpha_{i}^{ \pm}$as defined in Eq. (D6),

$$
\beta_{2}=\sqrt{4+y^{2}}, \quad \beta_{3}=\sqrt{4+9 y^{2}},
$$

and $x=\frac{\Lambda}{\mu}$ and $y=\frac{\Delta}{\mu}$, as defined in Eq. (66). 
[1] K. Rajagopal and F. Wilczek, arXiv:hep-ph/0011333.

[2] M. G. Alford, Ann. Rev. Nucl. Part. Sci. 51, 131 (2001).

[3] T. Schäfer, arXiv:hep-ph/0304281; hep-ph/0509068.

[4] D. H. Rischke, Prog. Part. Nucl. Phys. 52, 197 (2004).

[5] M. Buballa, Phys. Rept. 407, 205 (2005).

[6] H. c. Ren, arXiv:hep-ph/0404074.

[7] M. Huang, Int. J. Mod. Phys. E 14, 675 (2005).

[8] I. A. Shovkovy, Found. Phys. 35, 1309 (2005).

[9] D. D. Ivanenko and D. F. Kurdgelaidze, Astrophysics 1, 251 (1965) [Astrofiz. 1, 479 (1965)].

[10] N. Itoh, Prog. Theor. Phys. 44, 291 (1970).

[11] J. C. Collins and M. J. Perry, Phys. Rev. Lett. 34, 1353 (1975).

[12] M. G. Alford, K. Rajagopal, and F. Wilczek, Nucl. Phys. B 537, 443 (1999).

[13] I. A. Shovkovy and L. C. R. Wijewardhana, Phys. Lett. B 470, 189 (1999).

[14] T. Schäfer, Nucl. Phys. B 575, 269 (2000).

[15] N. J. Evans, J. Hormuzdiar, S. D. H. Hsu, and M. Schwetz, Nucl. Phys. B 581, 391 (2000).

[16] D. Nickel, R. Alkofer, and J. Wambach, Phys. Rev. D 74, 114015 (2006).

[17] T. Schäfer, Phys. Rev. D 65, 094033 (2002).

[18] R. Rapp, T. Schäfer, E. V. Shuryak, and M. Velkovsky, Annals Phys. 280, 35 (2000).

[19] I. A. Shovkovy and P. J. Ellis, Phys. Rev. C 66, 015802 (2002).

[20] C. Manuel, A. Dobado, and F. J. Llanes-Estrada, JHEP 0509, 076 (2005).

[21] M. G. Alford, M. Braby, S. Reddy, and T. Schäfer, Phys. Rev. C 75, 055209 (2007).

[22] R. Casalbuoni and R. Gatto, Phys. Lett. B 464, 111 (1999).

[23] D. T. Son and M. A. Stephanov, Phys. Rev. D 61 (2000) 074012; erratum ibid D 62, 059902 (2000).

[24] T. Schäfer, Phys. Rev. Lett. 85, 5531 (2000).

[25] R. Casalbuoni, R. Gatto, and G. Nardulli, Phys. Lett. B 498, 179 (2001) [Erratum-ibid. B 517, 483 (2001)].

[26] P. F. Bedaque and T. Schäfer, Nucl. Phys. A 697802 (2002).

[27] D. B. Kaplan and S. Reddy, Phys. Rev. D 65, 054042 (2002).

[28] N. Yamamoto, M. Tachibana, T. Hatsuda, and G. Baym, arXiv:0704.2654 [hep-ph].

[29] D. K. Hong, Phys. Lett. B 473, 118 (2000); Nucl. Phys. B 582, 451 (2000).
[30] S. R. Beane, P. F. Bedaque, and M. J. Savage, Phys. Lett. B 483, 131 (2000).

[31] G. Nardulli, Riv. Nuovo Cim. 25N3, 1 (2002).

[32] M. Rho, A. Wirzba, and I. Zahed, Phys. Lett. B 473 (2000) 126; M. Rho, E. V. Shuryak, A. Wirzba, and I. Zahed, Nucl. Phys. A 676 (2000) 273.

[33] D. K. Hong, T. Lee, and D. P. Min, Phys. Lett. B 477, 137 (2000).

[34] C. Manuel and M. H. G. Tytgat, Phys. Lett. B 479, 190 (2000).

[35] V. A. Miransky, I. A. Shovkovy, and L. C. R. Wijewardhana, Phys. Rev. D 63, 056005 (2001).

[36] Y. Nambu and G. Jona-Lasinio, Phys. Rev. 122, 345 (1961); Phys. Rev. 124, 246 (1961).

[37] U. Vogl and W. Weise, Prog. Part. Nucl. Phys. 27, 195 (1991).

[38] S. P. Klevansky, Rev. Mod. Phys. 64 (1992) 649.

[39] T. Hatsuda and T. Kunihiro, Phys. Rept. 247, 221 (1994).

[40] M. Buballa, Phys. Lett. B 609, 57 (2005).

[41] M. M. Forbes, Phys. Rev. D 72, 094032 (2005).

[42] H. J. Warringa, arXiv:hep-ph/0606063.

[43] K. Fukushima and K. Iida, Phys. Rev. D 71, 074011 (2005).

[44] D. Ebert and K. G. Klimenko, Phys. Rev. D 75, 045005 (2007); D. Ebert, K. G. Klimenko, and V. L. Yudichev, arXiv:0705.2666 [hep-ph].

[45] D. Blaschke, D. Ebert, K. G. Klimenko, M. K. Volkov, and V. L. Yudichev, Phys. Rev. D 70, 014006 (2004);

[46] D. Ebert, K. G. Klimenko, and V. L. Yudichev, Phys. Rev. C 72, 015201 (2005); D. Ebert, K. G. Klimenko, and V. L. Yudichev, Phys. Rev. D 72, 056007 (2005).

[47] A. W. Steiner, S. Reddy, and M. Prakash, Phys. Rev. D 66, 094007 (2002).

[48] S. B. Rüster, V. Werth, M. Buballa, I. A. Shovkovy, and D. H. Rischke, Phys. Rev. D 72, 034004 (2005).

[49] D. Blaschke, S. Fredriksson, H. Grigorian, A. M. Öztas, and F. Sandin, Phys. Rev. D 72, 065020 (2005).

[50] H. Abuki and T. Kunihiro, Nucl. Phys. A 768 (2006) 118.

[51] K. Rajagopal and F. Wilczek, Phys. Rev. Lett. 86, 3492 (2001).

[52] M. Ruggieri, arXiv:0705.2974 [hep-ph].

[53] T. Schäfer, Phys. Rev. D 65, 074006 (2002).

[54] D. Nickel and J. Wambach, in preparation. 